Collection SFN 7 (2007) 13-40

(c) EDP Sciences, Les Ulis

DOI: $10.1051 /$ sfn:2007017

\title{
La technique classique du rapport de flipping. Application aux aimants moléculaires et aux aimants photo-commutables
}

\author{
B. Gillon ${ }^{1}$
}

\author{
${ }^{1}$ Laboratoire Léon Brillouin (CEA-CNRS), CE Saclay, 91191 Gif-sur-Yvette, France
}

\begin{abstract}
Résumé. Des exemples d'applications de la méthode classique du rapport de flipping à la détermination de la densité de spin par diffraction de neutrons polarisés (DNP) sont décrits dans ce chapitre. Dans le domaine des composés magnétiques à base moléculaire, de telles investigations fournissent des informations directes sur la nature et l'origine des interactions intra- et inter-moléculaires. Ainsi, la visualisation de la distribution de spin dans la maille cristalline et dans la molécule permet de mettre en évidence les chemins d'interaction entre les centres magnétiques (ions de transition, radicaux organiques) et d'étudier le rôle des effets de délocalisation de spin et de polarisation de spin dans les mécanismes d'interaction. De plus, la densité de spin expérimentale dans des clusters moléculaires permet d'élucider la nature de l'état fondamental magnétique qui peut résulter de la compétition entre des interactions antiferromagnétiques intramoléculaires. Le photomagnétisme dans les composés à base moléculaire suscite un interêt croissant, en raison des applications potentielles des matériaux magnétiques photo-commutables. Les composés à transition de spin contenant un ion $\mathrm{Fe}^{2+}$ en coordination octaédrique présentent un état fondamental bas spin diamagnétique $(S=0)$ qui peut être commuté, sous irradiation lumineuse de longueur d'onde adéquate, vers un état métastable paramagnétique haut spin $(S=2)$ ayant une très longue durée de vie à basse température. La densité d'aimantation dans un état photo-excité magnétique a été déterminée pour la première fois pour le complexe à transition de spin $\left[\mathrm{Fe}^{\mathrm{II}}(\mathrm{ptz})_{6}\right]\left(\mathrm{BF}_{4}\right)_{2}(\mathrm{ptz}=1$-propyltetrazole $)$.
\end{abstract}

\section{INTRODUCTION}

Ce chapitre est consacré à l'utilisation de la technique classique du rapport de flipping pour des études de densité de spin dans des composés magnétiques qui sont "non classiques" à cause de leur caractère moléculaire, par contraste avec les composés intermétalliques habituels de métaux de transition ou de terres rares. De plus, certains de ces composés moléculaires ont des propriétés tout à fait particulières de photo-magnétisme, c'est à dire de présenter un magnétisme induit par irradiation à une lumière de longueur d'onde adaptée.

Les matériaux magnétiques à base moléculaire [1] peuvent être soit des cristaux moléculaires dans lesquels les interactions magnétiques entre molécules peuvent conduire à un ordre magnétique, soit des réseaux cristallins, obtenus en reliant des ions de métaux de transition par des précurseurs moléculaires, susceptibles de montrer une aimantation spontanée à la température ambiante, comme le dérivé du Bleu de Prusse $\mathrm{V}_{3}^{\mathrm{II}}\left[\mathrm{Cr}^{\mathrm{III}}(\mathrm{CN})_{6}\right]_{2} \cdot \mathrm{nH}_{2} \mathrm{O}$ [2]. Il existe une grande variété de comportements magnétiques dans les systèmes où les ions de transition sont couplés par des ponts organiques. Par exemple, les clusters moléculaires magnétiques, qui sont en fait des molécules magnétiques de taille nanométrique, peuvent présenter un comportement classique, tout comme un comportement quantique, car leur taille est intermédiaire entre celle des simples molécules magnétiques et celle du matériau massif [3].

Dans ce chapitre, nous allons d'abord rappeler les principes de la technique du rapport de flipping. Puis, nous présenterons les différentes méthodes d'analyse des données pour construire la densité d'aimantation à partir des rapports de flipping mesurés.

Nous introduirons les concepts de base concernant les mécanismes d'interaction magnétique dans les composés moléculaires. L'étude des densités de spin donne accès à des informations cruciales concernant les processus principaux d'interaction car elles permettent de déterminer la distribution des moments magnétiques dans la molécule (effet de délocalisation de spin), le signe de la densité de spin (effet de polarisation de spin) et la forme des densités atomiques (orthogonalité accidentelle) [4]. 
Pour illustrer l'intérêt de ces études, nous donnerons un certain nombre d'exemples de composés présentant des comportements magnétiques variés. Les premiers exemples concerneront des systèmes où le magnétisme provient des électrons non appariés $2 p$ de radicaux organiques; les exemples suivants montreront des systèmes où interagissent des ions de métaux de transition.

Enfin, nous décrirons la première détermination de la densité d'aimantation d'un état photo-excité d'un complexe $\mathrm{Fe}^{\mathrm{III}}$ à transition de spin.

\section{LA TECHNIQUE CLASSIQUE DU RAPPORT DE FLIPPING}

La technique classique du rapport de flipping consiste à mesurer les rapports de flipping des réflexions de Bragg lorsqu'un faisceau de neutrons polarisés est diffracté par un monocristal, de façon à obtenir une détermination expérimentale précise des facteurs de structures magnétiques.

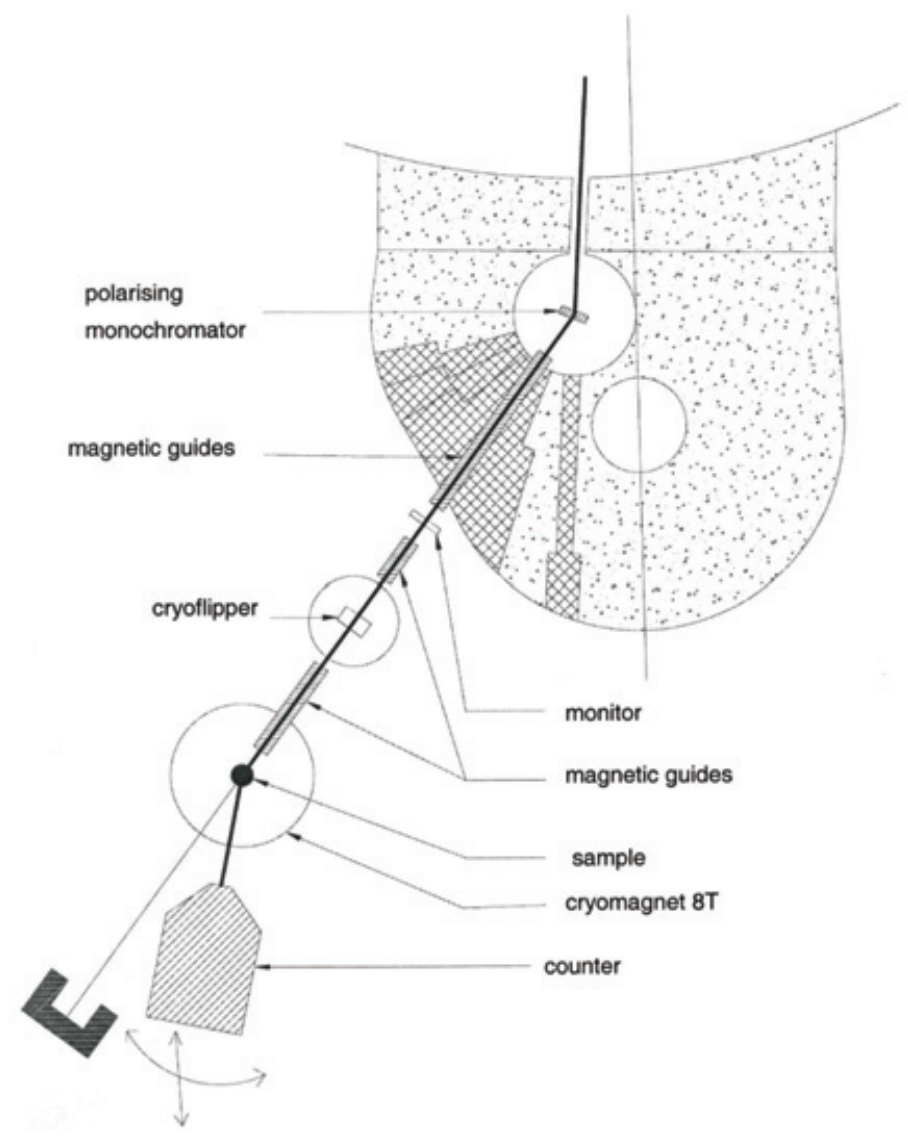

Figure 1. Le diffractomètre 5C1 au LLB.

Le diffractomètre $5 \mathrm{C} 1$ du LLB est un diffractomètre deux axes à neutrons polarisés avec un bras levant, placé sur un canal de la source chaude du réacteur Orphée (Figure 1). Il existe deux autres diffractomètres du même type à l'ILL, mais avec des caractéristiques un peu différentes: D3 sur la source chaude et D23 sur un guide thermique. 
Sur le diffractomètre $5 \mathrm{C} 1$, le faiscau monochromatique de neutrons polarisés est obtenu par diffraction sur un monocristal ferromagnétique d'Heusler, $\mathrm{Cu}_{2} \mathrm{MnAl}$, sur la réflexion (111) en géométrie de transmission. Le monochromateur est placé dans le champ magnétique statique $(0,4$ Tesla) d'un aimant permanent. La longueur d'onde utilisée, $0.84 \AA$, correspond au flux maximum de la source chaude et permet l'étude d'un large domaine du réseau réciproque. La polarisation du faisceau atteint $90 \%$. Cette polarisation est maintenue et guidée par un guide magnétique de 200 Gauss. La direction de cette polarisation peut être inversée; elle est contrôlée par un dispositif cryogénique de flipping. Un filtre d'erbium, idéal pour une longueur d'onde de $0.84 \AA$, supprime la contamination $\lambda / 2$. L'échantillon est placé dans un cryoaimant supraconducteur qui peut fournir 8 Tesla et refroidir le cristal échantillon jusqu'à 1,5 K. Le bras compteur peut s'abaisser en dessous ou s'élever au dessus du plan horizontal. Son domaine angulaire va de $-5^{\circ}$ à $+20^{\circ}$. Le programme de pilotage utilise le système Windows NT sur un PC.

Le champ magnétique vertical du cryoaimant aligne les moments magnétiques électroniques du cristal échantillon. En général, un champ de plusieurs Tesla permet d'induire une réponse assez forte dans le cas de cristaux paramagnétiques. Pour les cristaux ferromagnétiques, il suffit d'aligner les domaines magnétiques et le champ nécessaire est plus faible. Cependant, sur 5C1, il faut un champ minimum de 0,5 Tesla pour maintenir la polarisation du faisceau le long de sa trajectoire.

\subsection{Principes}

Les sections efficaces différentielles pour un faisceau de neutrons polarisés diffracté par une réflexion de Bragg d'un monocristal s'écrivent en terme de facteurs de structure nucléaire et magnétique :

$$
\begin{aligned}
& \left(\frac{d \sigma}{d \Omega}\right)^{ \pm \pm} \propto\left|F_{N} \pm F_{M}^{\perp z}\right|^{2} \\
& \left(\frac{d \sigma}{d \Omega}\right)^{ \pm \mp} \propto\left|F_{M}^{\perp x} \pm i F_{M}^{\perp y}\right|^{2}
\end{aligned}
$$

où $F_{N}(\mathbf{Q})$ est le facteur de structure nucléaire correspondant au vecteur de diffusion $\mathbf{Q}$ :

$$
F_{N}(\mathbf{Q})=\sum_{i}^{\text {atoms }} b_{i} e^{i \mathbf{Q} \cdot \mathbf{r}_{\mathbf{i}}} e^{-W_{i}}
$$

$b_{i}$ étant la longueur de diffusion des atomes $i$, caractéristique de l'élément chimique, $W_{i}$ un terme qui rend compte des vibrations des noyaux, et où le vecteur $\mathbf{F}_{\mathbf{M}}(\mathbf{Q})$ est le facteur de structure magnétique, composante de Fourier de la distribution magnétique $\mathbf{M}(\mathbf{r})$ :

$$
\mathbf{F}_{\mathbf{M}}(\mathbf{Q})=\int_{\text {unit cell }} \mathbf{M}(\mathbf{r}) e^{i \mathbf{Q} \cdot \mathbf{r}_{\mathbf{i}}} d \mathbf{r}
$$

Comme il n'y a pas d'analyse de la polarisation du faisceau diffracté, les intensités de ces faisceaux, pour les directions de polarisation incidentes 'up' et 'down', sont respectivement les sommes :

$$
\begin{aligned}
& I_{+}=\left(\frac{d \sigma}{d \Omega}\right)^{++}+\left(\frac{d \sigma}{d \Omega}\right)^{+-} \\
& I_{-}=\left(\frac{d \sigma}{d \Omega}\right)^{--}+\left(\frac{d \sigma}{d \Omega}\right)^{-+}
\end{aligned}
$$

ce qui conduit à l'expression :

$$
I(\mathbf{Q})_{ \pm} \propto\left[F_{N}^{2} \pm\left(F_{N}^{\star} F_{M}^{\perp z}+F_{N} F_{M}^{\perp z^{\star}}\right)+{F_{M}^{\perp}}^{2}\right]
$$


Le vecteur $F_{M}^{\perp}$ est la composante du facteur de structure magnétique perpendiculaire au vecteur de diffusion Q, et le terme $F_{M}^{\perp z}$ qui apparait dans les expressions (2.1), (2.2) et (2.7) en est sa composante verticale (parallèle au champ), comme représenté sur la Figure 2. Il peut s'écrire :

$$
F_{M}^{\perp z}=q F_{M}^{\perp}=q^{2} F_{M}
$$

où $q=\sin \alpha, \alpha$ étant l'angle entre le vecteur de diffusion $\mathbf{Q}$ et la direction du facteur de structure magnétique $\mathbf{F}_{\mathbf{M}}(\mathbf{Q})$.

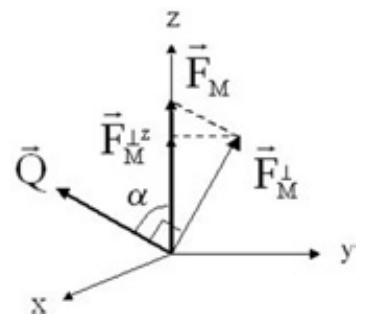

Figure 2. Définition géométrique de la composante $F_{M}^{\perp z}$.

A partir de (2.8) on obtient les expressions suivantes :

$$
I(\mathbf{Q})_{ \pm} \propto\left[F_{N}^{2} \pm q^{2}\left(F_{N}^{\star} F_{M}+F_{N} F_{M}^{\star}\right)+q^{2} F_{M}^{2}\right]
$$

Le rapport de flipping d'une réflexion de Bragg est le rapport entre les intensités diffractées pour les deux polarisations du faisceau incident :

$$
R(\mathbf{Q})=\frac{I_{+}}{I_{-}}
$$

L'avantage de mesurer un rapport comme celui-ci est que l'on s'affranchit de la mesure d'intensités intégrées. A la place, on mesure, pour chacune des deux polarisations incidentes, l'intensité au sommet de la réflexion et l'intensité du bruit de fond, de part et d'autre du pic :

$$
R(\mathbf{Q})=\frac{(\text { peak }- \text { background })_{+}}{(\text {peak }- \text { background })_{-}}
$$

Corrections dues aux imperfections du faisceau

Les expressions (2.9) et (2.10) supposent un faisceau parfaitement polarisé ainsi qu'une efficacité de flipping de 100\%. En fait la polarisation du faisceau incident est $P$ et l'efficacité du flipping est $e$, ces deux quantités étant inférieures à l'unité, avec :

$$
P=\frac{n_{+}+n_{-}}{n_{+}-n_{-}}
$$

L'expression du rapport de flipping est alors :

$$
R(\mathbf{Q})=\frac{F_{N}^{2}+P q^{2}\left(F_{N}^{\star} F_{M}+F_{N} F_{M}^{\star}\right)+q^{2} F_{M}^{2}}{F_{N}^{2}-P e q^{2}\left(F_{N}^{\star} F_{M}+F_{N} F_{M}^{\star}\right)+q^{2} F_{M}^{2}}
$$

Une troisième correction prend en compte la contamination $\lambda / 2$ résiduelle du faisceau incident, en ajoutant un terme $C_{+}(\lambda / 2)$ au numérateur et un terme $C_{-}(\lambda / 2)$ au dénominateur de l'expression (2.13). 


\section{Corrections dues à l'échantillon}

Un des avantages de la mesure d'un rapport d'intensités est qu'il n'y a pas à faire de corrections d'absorption. Cependant, d'autres corrections dues à l'échantillon lui-même sont à faire :

- Si l'échantillon a une très forte anisotropie magnétique, il peut y avoir dépolarisation à la surface de l'échantillon. La polarisation doit alors être corrigée par un facteur que l'on détermine en mesurant la polarisation devant et derrière le cristal.

- L'extinction agit différemment sur $I_{+}$et $I_{-}$. Le facteur de réduction $y_{ \pm}$dû à l'extinction dépend du pouvoir de diffraction $\sigma_{ \pm}$, qui n'est pas le même pour les polarisations 'up' et 'down', et en général il faut en tenir compte [5] :

$$
R_{e x t}=R\left(\frac{y_{+}}{y_{-}}\right)
$$

Cependant, dans le cas des cristaux moléculaires qui ne présentent que de faibles extinctions, celle-ci est habituellement négligée.

\section{Contributions de la polarisation nucléaire}

Dans les expressions des sections efficaces (2.1) et (2.2), n'ont été considérées pour l'interaction nucléaire que l'interaction indépendante du spin, et pour l'interaction magnétique que l'interaction dipôle-dipôle entre le moment magnétique du neutron et les moments magnétiques électroniques des couches non appariées.

Il existe en fait deux autres interactions qui entraînent des termes supplémentaires dans les expressions des sections efficaces :

- le couplage entre le spin du neutron $\boldsymbol{\sigma}$ et le spin nucléaire $\mathbf{I}_{\mathbf{i}}$ résultant en une partie de l'opérateur interaction nucléaire qui dépend du spin :

$$
\widehat{b}_{i}=b_{i}+B_{i} \mathbf{I}_{\mathbf{i}} \boldsymbol{\sigma}
$$

Ce terme dépend de la polarisation des neutrons incidents et est proportionnel à la polarisation des spins nucléaires. Pour les conditions expérimentales habituelles, il est en général très petit. Le coefficient $B_{i}$ est donné par :

$$
B_{i}=\frac{1}{2 I_{i}+1}\left(b_{i}^{+}-b_{i}^{-}\right)
$$

Or il se trouve que ce coefficient est particulièrement important pour l'hydrogène. Aussi, la contribution qui résulte de la polarisation des spins nucléaires de l'hydrogène dans un champ magnétique fort peut ne pas être négligeable pour un composé organique ou organo-métallique, à cause du grand nombre d'atomes d'hydrogène dans la maille. On peut écrire le facteur de structure de polarisation nucléaire de la façon suivante :

$$
F_{\mathrm{NP}}(\mathbf{Q})=\sum_{i}^{\mathrm{H} \text { atoms }} f_{\mathrm{NP}}^{i} e^{i \mathbf{Q} \cdot \mathbf{r}_{\mathbf{i}}}
$$

où le coefficient de polarisation $f_{\mathrm{NP}}^{i}$ est égal à :

$$
f_{\mathrm{NP}}^{i}=14.89 \times 10^{-4} \frac{\mathrm{H}(\text { Tesla })}{\mathrm{T}(\mathrm{K})}\left(10^{-12} \mathrm{~cm}\right)
$$

- l'interaction entre les moments magnétiques des neutrons et le champ électrique crée par l'ensemble des électrons et des noyaux [6].

Cet effet est appelé l'effet Schwinger. Le facteur de structure d'effet Schwinger $\left(A_{S}+i B_{S}\right)$ est calculé en utilisant les facteurs de forme électronique [7,8]. 


\subsection{Le cas des structures centro-symétriques}

Pour les structures centro-symétriques, les deux facteurs de structure, nucléaire et magnétique, sont réels. L'expression (2.13) pour le rapport de flipping devient alors :

$$
R(\mathbf{Q})=\frac{F_{N}^{2}+2 P q^{2} F_{N} F_{M}+q^{2} F_{M}^{2}}{F_{N}^{2}-2 P e q^{2} F_{N} F_{M}+q^{2} F_{M}^{2}}
$$

La mesure du rapport de flipping expérimental $R_{\exp }$ permet d'écrire une équation du deuxième degré :

$$
\gamma^{2}+B \gamma+C=0
$$

avec comme variable :

$$
\gamma=\left(\frac{F_{M}}{F_{N}}\right)
$$

et des coefficients $B$ et $C$ qui sont connus à partir des données expérimentales :

$$
\begin{gathered}
B=-2 P\left(\frac{R_{\exp } e+1}{R_{\exp }-1}\right) \\
C=\frac{1}{q^{2}}
\end{gathered}
$$

Le choix entre les deux racines de cette équation du deuxième degré est en général évident. Il permet d'obtenir la valeur expérimentale $\gamma^{\exp }$ du rapport entre les facteurs de structure magnétique et nucléaire. Le facteur de structure magnétique est alors donné par :

$$
F_{M}^{e x p}=\gamma^{\exp } F_{N}
$$

Pour cela, on utilise pour $F_{N}$ soit le facteur de structure nucléaire calculé avec les positions et les coefficients d'agitation thermique correspondant à la température où l'expérience de neutrons polarisés a eu lieu, soit la racine carrée de l'intensité intégrée mesurée à cette même température $\sqrt{F_{N}^{2}}$, avec son signe théorique.

Contribution due à la polarisation des spins nucléaires de l'hydrogène

Le terme $F_{N P}(\mathbf{Q})$ dépend de la polarisation des neutrons. Dans le cas centro-symétrique, la section efficace (2.7) en tient compte de la façon suivante :

$$
I(\mathbf{Q})_{ \pm} \propto\left[F_{N}^{2} \pm 2 F_{N}\left(F_{M}^{\perp z}+F_{N P}\right)+{F_{M}^{\perp}}^{2}+F_{N P}^{2}+2 F_{M}^{\perp} F_{N P}\right]
$$

Le rapport de flipping s'écrit ainsi :

$$
R(\mathbf{Q})=\frac{F_{N}^{2}+2 P F_{N}\left(q^{2} F_{M}+F_{N P}\right)+q^{2} F_{M}^{2}+F_{N P}^{2}+2 q F_{M} F_{N P}}{F_{N}^{2}-2 P e F_{N}\left(q^{2} F_{M}+F_{N P}\right)+q^{2} F_{M}^{2}+F_{N P}^{2}+2 q F_{M} F_{N P}}
$$

et les coefficients de l'équation du deuxième degré (2.20) doivent être remplacés par :

$$
\begin{gathered}
B=-2 P\left(\frac{R_{\exp } e+1}{R_{\exp }-1}\right)+2\left(\frac{F_{N P}}{F_{N}}\right) \\
C=\frac{1}{q^{2}}-\frac{2 P}{q^{2}}\left(\frac{R_{\exp } e+1}{R_{\exp }-1}\right) \frac{F_{N P}}{F_{N}}+\frac{1}{q^{2}}\left(\frac{F_{N P}}{F_{N}}\right)^{2}
\end{gathered}
$$




\section{Contribution orbitale}

La densité d'aimantation est la somme d'une contribution de spin due à la présence des électrons non appariés et d'une contribution d'orbite due à leur mouvement orbital autour du noyau.

$$
M(\mathbf{r})=\rho_{S}(\mathbf{r})+\rho_{L}(\mathbf{r})
$$

Le facteur de structure magnétique est lui aussi la somme de deux contributions :

$$
F_{M}(\mathbf{Q})=F_{M}^{S}(\mathbf{Q})+F_{M}^{L}(\mathbf{Q})
$$

Pour les radicaux organiques, les électrons non appariés se meuvent sur une orbitale $2 p$, mais cette orbitale $2 p$ est bloquée par la liaison chimique. La densité d'aimantation se réduit alors à la densité de spin. Pour les systèmes contenant des ions métalliques, il faut prendre en compte la contribution orbitale, sauf pour le cas des ions avec un moment angulaire nul comme l'ion $\mathrm{Mn}^{2+}$ ion avec sa couche $3 \mathrm{~d}$ à demi remplie $\left(3 d^{5}, e_{g}^{2} t_{2 g}^{3}, S=\frac{5}{2}\right)$ ou l'ion $\mathrm{Cr}^{3+}\left(3 d^{3}, t_{2 g}^{3}, S=\frac{3}{2}\right)$. Cependant pour la plupart des ions de transition, le couplage spin-orbite est bien plus petit que le champ cristallin et le moment orbital est presque entièrement bloqué. La contribution orbitale peut alors être soit négligée, soit traitée comme une correction en utilisant l'approximation dipolaire [9] :

$$
F_{M}^{L}(\mathbf{Q})=m_{s} \frac{(g-2)}{g}\left(\left\langle j_{0}\right\rangle+\left\langle j_{2}\right\rangle\right)
$$

où $m_{s}$ est le moment magnétique associé au spin, $g$ le facteur de Landé et où $\left\langle j_{0}\right\rangle,\left\langle j_{2}\right\rangle$ sont les intégrales radiales, transformées de Fourier des fonctions radiales à l'aide des fonctions de Bessel sphériques respectivement d'ordre 0 et 2 [10].

La partie spin pur du facteur de structure se déduit alors du facteur de structure magnétique expérimental par la soustraction.

$$
F_{M}^{S}(\mathbf{Q})=F_{M}^{\exp }(\mathbf{Q})-F_{M}^{L}(\mathbf{Q})
$$

\subsection{Cas non centro-symétrique}

Dans le cas d'un groupe d'espace non centro-symétrique, les deux facteurs de structure nucléaire et magnétique sont des quantités complexes, avec des parties réelles $A_{N}, A_{M}$ et des parties imaginaires $B_{N}, B_{M}$. L'expression (2.13) du rapport de flipping devient :

$$
R(\mathbf{Q})=\frac{A_{N}^{2}+B_{N}^{2}+2 P q^{2}\left(A_{N} A_{M}+B_{N} B_{M}\right)+q^{2}\left(A_{M}^{2}+B_{M}^{2}\right)}{A_{N}^{2}+B_{N}^{2}-2 P e q^{2}\left(A_{N} A_{M}+B_{N} B_{M}\right)+q^{2}\left(A_{M}^{2}+B_{M}^{2}\right)}
$$

A la différence du cas centro-symétrique, il n'est pas possible, pour chaque réflexion de Bragg, de déduire les deux quantités inconnues $A_{M}$ et $B_{M}$ à partir de la mesure du seul rapport de flipping. Pour contourner cette difficulté, une modélisation paramétrique de la densité de spin est utilisée, qui permet d'affiner les paramètres du modèle en comparant les rapports de flipping calculés par le modèle aux rapports de flipping expérimentaux [11]. Pour ce faire, toutes les corrections sont introduites dans l'expression (2.33). La formule générale donnant le rapport de flipping dans le cas non centrosymétrique et prenant en considération la polarisation des spins nucléaires $\left(A_{P N}+i B_{P N}\right)$ et la correction d'effet Schwinger $\left(A_{S}+i B_{S}\right)$, est alors :

$$
R(\mathbf{Q})=\frac{A_{N}^{2}+B_{N}^{2}+2 P A+q^{2} B+\left(A_{P N}-B_{S}\right)^{2}+\left(B_{P N}+A_{S}\right)^{2}+C_{+}}{A_{N}^{2}+B_{N}^{2}-2 P e A+q^{2} B+\left(A_{P N}-B_{S}\right)^{2}+\left(B_{P N}+A_{S}\right)^{2}+C_{-}}
$$


dans lequel

$$
\begin{aligned}
& A=A_{N}\left(q^{2} A_{M}+A_{P N}-B_{S}\right)+B_{N}\left(q^{2} B_{M}+B_{P N}-A_{S}\right) \\
& B=A_{M}^{2}+B_{M}^{2}+2\left(\left(A_{P N}-B_{S}\right) A_{M}+\left(B_{P N}-A_{S}\right) B_{M}\right)
\end{aligned}
$$

et où $C_{ \pm}$est la correction due à la contamination $(\lambda / 2)$.

\section{RECONSTRUCTION DE LA DENSITÉ DE SPIN}

A partir des facteurs de structure magnétiques déduits de l'expérience (ou des rapports de flipping dans les cas non centro-symétriques), on peut reconstruire la densité de spin, soit directement sans aucun modèle, soit en affinant les paramètres d'un modèle de façon à satisfaire les données expérimentales.

\subsection{Reconstruction directe de la densité de spin (sans modèle paramétrique)}

Il y a deux façons possibles pour retrouver la densité de spin à partir des données expérimentales (les $F_{M}$ ou les $R$ ) sans faire aucune supposition sur la nature de cette distribution : la somme de Fourier inverse (qui ne s'applique que dans le cas centro-symétrique où les $F_{M}$ peuvent être déterminés) et la méthode du Maximum d'Entropie.

\section{Somme de Fourier inverse}

La densité de spin peut s'écrire comme la transformée de Fourier inverse des facteurs de structure magnétiques expérimentaux, à condition que la somme soit infinie :

$$
\rho_{S}(\mathbf{r})=\frac{1}{V} \sum_{\mathbf{Q}}^{\infty} F_{M}^{S}(\mathbf{Q}) e^{-i \mathbf{Q} . \mathbf{r}}
$$

où $V$ est le volume de la maille.

En pratique, cette somme est limitée par la limite expérimentale de la quantité $\sin \theta / \lambda$. De plus, seules les réflexions de Bragg pour lesquelles le facteur de structure nucléaire $F_{N}$ est suffisant peuvent être mesurées, quelle que soit la valeur du facteur de structure $F_{M}$. Aussi l'absence des réflexions correspondant aux faibles $F_{N}$ dans la somme (3.1) peut entraîner des biais importants de la densité ainsi reconstruite.

\section{Maximum d'Entropie}

La première application de la Méthode du Maximum d'Entropie (MaxEnt) à la reconstruction de la densité de spin a été proposée par Papoular et al. [12].

La densité d'aimantation $\rho(\mathbf{r})$ reconstruite par MaxEnt est la distribution qui maximise l'entropie et en même temps remplit la condition $\chi^{2}=1$. Pour calculer l'entropie, la maille du composé est quadrillée en $\mathrm{M}$ pixels dans lesquels on suppose la densité constante. Cependant, comme la densité de spin peut être aussi bien négative que positive et que l'entropie n'est définie que pour des quantités positives, on considère une double distribution de quantités positives $n_{i}=\rho^{+}\left(\mathbf{r}_{\mathbf{i}}\right)$ et $n_{i+M}=\rho^{-}\left(\mathbf{r}_{\mathbf{i}}\right)$, la densité d'aimantation étant donnée par $\rho_{i}=\rho^{+}\left(\mathbf{r}_{\mathbf{i}}\right)-\rho^{-}\left(\mathbf{r}_{\mathbf{i}}\right)$. L'entropie de la densité Dans l'd'aimantation est alors définie par :

$$
S(\rho)=-\sum_{i=1}^{2 M} p_{i} \log \left(p_{i}\right)
$$

où $p_{i}$ est la densité normalisée au pixel $i$ :

$$
p_{i}=\frac{\rho_{i}}{\sum_{j=1}^{2 M} \rho_{j}}
$$


et l'expression de $\chi^{2}$ pour un jeu de $N$ facteurs de structures expérimentaux est :

$$
\chi^{2}(\rho)=\frac{1}{N} \sum_{\mathbf{Q}} \frac{\left|F_{M}^{\exp }(\mathbf{Q})-F_{M}^{\mathrm{calc}, \rho}(\mathbf{Q})\right|^{2}}{\sigma^{2}(\mathbf{Q})}
$$

où $F_{M}^{c a l c, \rho}(\mathbf{Q})$ est le facteur de structure magnétique calculé pour la distribution d'aimantation définie plus haut. La méthode du Maximum d'Entropie a ensuite été généralisée aux cas non centro-symétriques [13].

\subsection{Affinement d'un modèle paramétrique}

On peut considérer différentes approches selon la grandeur que l'on modélise : la fonction d'onde de l'électron non apparié ou la densité de spin [14]. Dans les deux cas, le modèle consiste en une expression analytique, avec des paramètres que l'on affine en comparant les facteurs de structure magnétiques théoriques (ou les rapports de flipping dans les cas non centro) aux valeurs expérimentales. Ces paramètres servent ensuite à reconstruire la densité de spin avec le modèle analytique.

\section{Modèle de la fonction d'onde}

Dans la description Hartree-Fock Restrainte (Restricted Hartree-Fock ou RHF) de la fonction d'onde d'un système moléculaire avec un électron non apparié, la densité de spin est donnée par le carré du module de l'orbitale moléculaire $\phi(\mathbf{r})$ occupée par un seul électron :

$$
\rho(\mathbf{r})=|\phi(\mathbf{r})|^{2}
$$

où l'orbitale moléculaire $\phi$ s'écrit comme une combinaison linéaire d'orbitales atomiques $\psi_{i}\left(\mathbf{r}_{\mathbf{i}}\right)$ centrées sur les atomes $i$ :

$$
\phi(\mathbf{r})=\sum_{i}^{\text {atomes }} \alpha_{i} \psi_{i}(\mathbf{r})
$$

Les orbitales atomiques sont elles-mêmes des combinaisons linéaires de fonctions atomiques de type Slater :

$$
\psi_{i}\left(\mathbf{r}_{\mathbf{i}}\right)=\sum_{L=0}^{N-1} R_{L}^{i}\left(\xi_{L}^{i}, r_{i}\right) \sum_{M=-L}^{L} a_{L M}^{i} Y_{L M}^{i}\left(\theta_{i}, \varphi_{i}\right)
$$

où $N, L$ et $M$ sont les nombres atomiques quantiques : $N$ est le premier nombre quantique $(N=0$ pour $1 s, N=1$ pour $2 s, 2 p ; N=2$ pour $3 s, 3 p, 3 d) ; L=0$ à $N-1$, le deuxième nombre quantique ( $L=0$ pour les orbitales $s ; L=1$ pour les orbitales $p$ et $L=2$ pour les orbitales $d$ ) et $M$ est le nombre quantique magnétique ( $M$ va de $-L$ à $L$ ). Les $Y_{L M}^{i}\left(\theta_{i}, \varphi_{i}\right)$ sont les harmoniques sphériques habituelles et les fonctions radiales sont les fonctions de Slater :

$$
R_{L}^{i}\left(\xi_{L}^{i}, r_{i}\right)=N_{r} r_{i}^{N} e^{-\xi_{L}^{i} r_{i}}
$$

Les coefficients $a_{L M}^{i}$ sont les coefficients des orbitales atomiques avec la condition de normalisation :

$$
\sum_{M=-L}^{L}\left(a_{L M}^{i}\right)^{2}=1
$$

A partir de l'expression (2.4), on peut écrire le facteur de structure magnétique comme :

$$
F_{M}(\mathbf{Q})=\int \phi(\mathbf{r})^{\star} e^{i \mathbf{Q} \cdot \mathbf{r}} \phi(\mathbf{r}) d \mathbf{r}
$$


Avec la description de l'orbitale moléculaire $\phi(\mathbf{r})$ comme une superposition d'orbitales atomiques (3.6), on aboutit à des termes impliquant des orbitales atomiques qui sont soit centrées sur le même atome (un centre) soit centrées sur différents atomes (deux centres). Si on néglige le recouvrement entre deux atomes voisins, l'expression de $F_{M}$ devient :

$$
F_{M}(\mathbf{Q})=\sum_{i}^{\text {atoms }} p_{i} f_{m}^{i}(\mathbf{Q}) e^{i \mathbf{Q} \cdot \mathbf{R}_{\mathbf{i}}} e^{-W_{i}}
$$

où $f_{m}^{i}(\mathbf{Q})$ est le facteur de forme magnétique normalisé de l'atome $i$, qui se trouve en position $\mathbf{R}_{\mathbf{i}}$, et qui porte une population de $\operatorname{spin} p_{i}$ :

$$
f_{m}^{i}(\mathbf{Q})=\int \psi_{i}(\mathbf{r})^{\star} e^{i \mathbf{Q} \cdot \mathbf{r}} \psi_{i}(\mathbf{r}) d \mathbf{r}
$$

Après développement de $f_{m}^{i}(\mathbf{Q})$, en utilisant l'expression (3.7) de l'orbitale atomique $\psi_{i}(\mathbf{r})$, on obtient l'expression suivante pour $F_{M}$ :

$$
F_{M}(\mathbf{Q})=\sum_{i}^{\text {atoms }} p_{i} \sum_{L=0}^{\infty}\left\langle j_{L}(\mathbf{Q})\right\rangle \sum_{M=-L}^{L} c_{L M} Y_{L M}^{\star}\left(\theta_{Q}, \varphi_{Q}\right) e^{i \mathbf{Q} \cdot \mathbf{R}_{\mathbf{i}}} e^{-W_{i}}
$$

où les $c_{L M}$ correspondent à des produits de coefficients orbitaux $a_{L_{1} M_{1}}^{i} a_{L_{2} M_{2}}^{i}$ via les coefficients $3 \mathrm{j}$ utilisés pour calculer les intégrales des produits de trois harmoniques sphériques [7] :

$$
\begin{aligned}
c_{L M}^{i}= & i^{L}(4 \pi)^{1 / 2}(2 L+1)^{1 / 2}\left(2 L_{1}+1\right)\left(\begin{array}{ccc}
L_{1} & L_{1} & L \\
0 & 0 & 0
\end{array}\right) \\
& \times \sum_{M_{1}, M_{2}}(-1)^{M}\left(\begin{array}{ccc}
L_{1} & L_{1} & L \\
-M_{1} & M_{2} & M
\end{array}\right) a_{L_{1} M_{1}}^{i \star} a_{L_{2} M_{2}}^{i}
\end{aligned}
$$

et où les $\left\langle j_{L}(\mathbf{Q})\right\rangle$ sont les transformées de Fourier-Bessel des fonctions orbitales radiales :

$$
\left\langle j_{L}(\mathbf{Q})\right\rangle=\int_{0}^{\infty} r^{2}\left(R_{L}^{W F}(r)\right)^{2} j_{L}(Q . r) d r
$$

les $j_{L}(Q r)$ étant les fonctions de Bessel sphériques.

Dans l'expression finale des facteurs de structure magnétiques (3.13), les paramètres à affiner sont les populations atomiques de spin $p_{i}$ et les coefficients orbitaux $a_{L M}^{i}$. Un programme d'affinement par moindres carrés a été écrit par E. Ressouche pour les orbitales $2 p$ [7].

\section{Modélisation multipolaire de la densité de spin}

Une modélisation multipolaire de la densité de spin (programme Molly) [15] a été dérivée du formalisme de Hansen et Coppens pour décrire la densité de charge [16]. La densité de spin est décrite comme une somme de densités atomiques :

$$
\rho(\mathbf{r})=\sum_{i} \rho_{i}^{\text {mult }}\left(\mathbf{r}_{\mathbf{i}}\right)
$$

où $\mathbf{r}_{\mathbf{i}}=\mathbf{r}-\mathbf{R}_{\mathbf{i}}$.

La densité de spin atomique est développée sur une base de fonctions multipolaires :

$$
\rho_{i}^{\text {mult }}\left(\mathbf{r}_{\mathbf{i}}\right)=P_{v}^{i} R_{0}^{i}\left(\kappa^{\prime} r_{i}\right)+\sum_{l=0}^{l_{\max }=4} R_{l}^{i}\left(\kappa^{\prime \prime} r_{i}\right) \sum_{m=-l}^{l} P_{l m}^{i} y_{l m}^{i}(\theta, \varphi)
$$


où la fonction radiale $R_{l}^{i}(\kappa r)$ est une fonction de Slater de variable $\kappa r$ ( $\kappa$ étant un coefficient de contraction qui peut être affiné)

$$
R_{l}^{i}(\kappa r)=\frac{\kappa^{3} \zeta_{l}^{n_{l}+3}}{\left(n_{l}+2\right) !} r_{i}^{n_{l}} e^{-\kappa \zeta_{l}^{i} r_{i}}
$$

et où les $y_{l m}^{i}\left(\theta_{i}, \varphi_{i}\right)$ sont les harmoniques sphériques réelles :

$$
\begin{aligned}
& y_{l m+}=\frac{1}{2}\left(Y_{l m+}+Y_{l m-}\right) \\
& y_{l m-}=\frac{1}{2 i}\left(Y_{l m+}-Y_{l m-}\right)
\end{aligned}
$$

Le plus souvent, on se cantonne au deuxième terme de (3.18). Le premier terme permet d'affiner une densité de spin qui est une somme de deux contributions venant d'orbitales différentes comme par exemple $2 s$ et $2 p$ dans un cas d'hybridation.

Les expressions du facteur de structure $F_{M}$ provenant de la transformée de Fourier du développement en multipôles (3.17) ressemble beaucoup à celui qui provient de la modélisation en fonctions d'onde, à ceci près que les harmoniques sphériques habituelles ont été remplacées par les harmoniques sphériques réelles

$$
F_{M}(\mathbf{Q})=\sum_{i}^{\text {atoms }} \sum_{l=0}^{4} i^{l}\left\langle j_{l}(\mathbf{Q})\right\rangle \sum_{m=-l}^{l} P_{l m} y_{l m}\left(\theta_{Q}, \varphi_{Q}\right) e^{i \mathbf{Q} \cdot \mathbf{R}_{\mathbf{i}}} e^{-W_{i}}
$$

et que les transformées de Fourier-Bessel $\left\langle j_{l}(\mathbf{Q})\right\rangle$ sont les transformées de la densité radiale des fonctions de Slater $R_{l}^{\text {dens }}(r)$, qui correspondent aux carrés des $R_{L}^{W F}(r)$ de la formule (3.8)) :

$$
\left\langle j_{l}(\mathbf{Q})\right\rangle=\int_{0}^{\infty} r^{2}\left(R_{l}^{\mathrm{dens}}(r)\right) j_{l}(Q r) d r
$$

Dans l'expression (3.22) du facteur de structure magnétique, les paramètres à affiner sont les coefficients multipolaires $P_{l m}^{i}$ et les coefficients de contraction radiale $\kappa$. Les valeurs initiales des exposants de Slater sont déduites des calculs de fonctions d'onde que l'on trouve dans la littérature [17], en prenant $\zeta^{i}=2 \xi^{i}$ dans la mesure où la densité de spin correspond au carré de la densité de l'orbitale atomique.

Une méthode de moindres carrés permet de déterminer ces paramètres en comparant les facteurs de structure magnétiques calculés aux facteurs de structure expérimentaux. Dans le cas des structures non centro-symétriques, ce sont les rapports de flipping que l'on compare, les rapports théoriques étant calculés à partir de (2.34) en utilisant l'expression (3.22) de $F_{M}(\mathbf{Q})$ qui est complexe dans ce cas, toutes les autres quantités étant introduites comme données. La densité de spin est ensuite reconstruite à partir de l'expression (3.18), avec les paramètres affinés $P_{l m}^{i}$ et $\kappa$.

\section{Modèle général}

Grâce à l' analogie entre les deux descriptions, il est possible de reconstruire la densité de spin en utilisant le modèle de fonction d'onde pour certains atomes et le modèle multipolaire pour les autres. Il faut noter que dans ce cas la fonction $\phi(\mathbf{r})$ de l'expression (3.5) n'est pas une orbitale moléculaire du système car elle est limitée à un certain nombre d'atomes. On suppose de plus que ce modèle peut s'appliquer à des systèmes où le nombre d'électrons non appariés est $n(n>1)$, alors que $\phi(\mathbf{r})$ aurait dû être remplacé dans (3.5) par un déterminent de Slater construit avec les orbitales moléculaires des $n$ électrons.

L'expression de la densité de spin est obtenue en remplaçant $\phi(\mathbf{r})$ par son développement en orbitales atomiques [18] :

$$
\rho(\mathbf{r})=\left|\sum_{i}^{a t o m s} \alpha_{i} \psi_{i}\left(\mathbf{r}_{\mathbf{i}}\right)\right|^{2}
$$


Si on néglige les termes croisés, cette expression devient :

$$
\rho(\mathbf{r})=\sum_{i}^{\text {atoms }} \alpha_{i}^{2}\left|\psi_{i}(\mathbf{r})\right|^{2}
$$

Pour permettre des densités négatives, le coefficient $\alpha_{i}^{2}$ est remplacé par un paramètre de population pi qui peut être positif ou négatif. Ceci correspond au modèle des orbitales atomiques :

$$
\rho_{i}^{a t}\left(\mathbf{r}_{\mathbf{i}}\right)=p_{i}\left|\psi_{i}\left(\mathbf{r}_{\mathbf{i}}\right)\right|^{2}
$$

Les orbitales atomiques ont une expression proche de (3.7), mais les harmoniques sphériques sont remplacées par des harmoniques sphériques réelles [18]:

$$
\psi_{i}\left(\mathbf{r}_{\mathbf{i}}\right)=R_{N, L}^{i}\left(\xi_{l}^{i}, r_{i}\right) \sum_{M=-L}^{L} a_{L M}^{i} N_{a n g} y_{L M}^{i}\left(\theta_{i}, \varphi_{i}\right)
$$

où les parties radiales des fonctions d'onde sont des fonctions de Slater et où les $a_{L M}^{i}$ sont les coefficients des orbitales atomiques, avec la condition de normalisation (3.9).

On peut développer la densité atomique de spin de la formule (3.26) à l'aide des relations suivantes :

- le produit de deux fonctions de Slater est une fonction de Slater :

$$
R_{N, L}^{i}\left(\xi_{L}^{i}, r_{i}\right) R_{N, L}^{i}\left(\xi_{L}^{i}, r_{i}\right)=\aleph^{2} r_{i}^{2 N} e^{-2 \xi_{L}^{i} r_{i}}
$$

- le produit de deux harmoniques sphériques est une somme d'harmoniques sphériques :

$$
y_{L M_{1}}^{i}(\theta, \varphi) y_{L M_{2}}^{i}(\theta, \varphi)=\sum_{l=1}^{l_{\max }} c_{l} y_{l, M_{1}+M_{2}}^{i}(\theta, \varphi)
$$

L'expression de la densité atomique prend finalement la forme :

$$
\rho_{i}^{a t}\left(\mathbf{r}_{\mathbf{i}}\right)=R_{2 N, L}^{i}\left(2 \xi_{L}^{i}, r_{i}\right) \sum_{l=1}^{l_{\max }} \sum_{m=-l}^{l} P_{l, m}^{i} y_{l, m}^{i}(\theta, \varphi)
$$

où le coefficient $P_{l m}^{i}$ est une combinaison linéaire des produits des coefficients atomiques.

L'expression (3.30) est semblable au deuxième terme du développement multipolaire (3.18) si on prend la même la fonction pour tous les multipôles de $l=1$ à $l_{\text {max }}$, avec les exposants $n_{l}=2 \mathrm{~N}$ et $\zeta_{l}^{i}=2 \xi_{L}^{i}$. Les relations entre les coefficients des orbitales atomiques $a_{L M}^{i}$ et les coefficients des multipôles $P_{l m}^{i}$ ont été établies pour les orbitales 2 p [11] et étendues aux orbitales 3d [7]. Elles ont été introduites comme des contraintes dans une version modifiée du programme Molly, ce qui permet d'affiner les coefficients des orbitales pour certains atomes (les atomes $i$ ) et d'obtenir ainsi les populations des multipôles correspondant (programme MOL3D [7]). En même temps, on peut affiner les coefficients des multipôles pour les atomes $i^{\prime}$, autres que les atomes $i$ :

$$
\rho(\mathbf{r})=\sum_{i} \rho_{i}^{a t}\left(\mathbf{r}_{\mathbf{i}}\right)+\sum_{i^{\prime}} \rho_{i^{\prime}}^{\text {mult }}\left(\mathbf{r}_{\mathbf{i}^{\prime}}\right)
$$

La densité de spin dans la molécule est ensuite reconstruite à partir des paramètres des multipôles de tous les atomes.

La bibliothèque CCSL [19] permet aussi d'effectuer des affinements de multipôles en utilisant des facteurs de forme calculés à partir de la partie radiale soit de la fonction d'onde, soit de la partie multipolaire. 


\section{MAGNÉTISME DE COMPOSÉS MOLÉCULAIRES}

Les composés magnétiques à base moléculaire sont des solides constitués de fragments magnétiques comme des radicaux organiques ou des complexes d'ions de transition (ou de terres rares). Deux effets sont caractéristiques de ce type de composés : la délocalisation de spin et la polarisation de spin, effets qui se propagent sur toute la molécule à partir d'un moment fort, localisé sur un atome. Le premier effet engendre une densité de spin de même signe sur les atomes voisins, tandis que la polarisation de spin induit une densité de signe contraire.

L'interaction magnétique entre deux atomes magnétiques, par exemple deux ions de transition couplés par un pont organique, peut être décrite comme l'interaction entre deux entités moléculaires $A$ et $B$ couplées par un superéchange magnétique ou par un transfert de charge dans le système $A B$ [1]. Une bonne approximation consiste à ne considérer que les électrons non appariés des orbitales magnétiques construites à partir des orbitales $d$ des ions métalliques et des orbitales des atomes des ligands organiques.

\subsection{Superéchange magnétique}

L'hamiltonien d'échange entre les spins localisés $S_{A}$ et $S_{B}$ peut s'écrire [20]:

$$
H=-J S_{A} S_{B}
$$

où $J$ est la constante de couplage magnétique. Dans le cas où chaque entité $A$ et $B$ ne comprend qu'un seul électron non apparié, sur des orbitales $a$ et $b$ respectivement, on peut écrire $J$ comme la somme de deux termes :

$$
J=K+2 \beta S
$$

où $K$ est l'intégrale d'échange à deux électrons impliquant les deux orbitales magnétiques $a$ et $b$ :

et où $\beta$ est :

$$
\begin{gathered}
K=\left\langle a(1) b(2)\left|\frac{e^{2}}{r_{12}}\right| a(2) b(1)\right\rangle \\
\beta=\langle a|h| b\rangle
\end{gathered}
$$

$h$ est l'hamiltonien monoélectronique qui prend en compte l'énergie cinétique de l'électron actif ainsi que son interaction avec les noyaux et avec les électrons "passifs". Enfin, $S$ est l'intégrale de recouvrement entre les orbitales magnétiques :

$$
S=\langle a \mid b\rangle
$$

Le premier terme, $K$, est toujours positif et favorise ainsi une interaction ferromagnétique. Au contraire, le produit $S \beta$ est toujours négatif (si $S$ n'est pas nul) et favorise une interaction antiferromagnétique. La constante de couplage peut donc être écrite comme la somme de deux interactions : une ferro et une antiferro :

$$
J=J_{F}+J_{A F}
$$

Le terme antiferromagnétique est proportionnel à l'intégrale de recouvrement $S$ et est d'autant plus fort qu'il y a un grand recouvrement entre les orbitales magnétiques, ce qui est le cas le plus fréquent dans la nature.

Les mécanismes principaux qui conduisent à une interaction ferromagnétique sont :

- une stricte orthogonalité entre les orbitales magnétiques pour des raisons de symétrie

- tune orthogonalité accidentelle (mécanisme de délocalisation de spin) due à la géométrie 
- une polarisation de spin (ou premier mécanisme de Mc Connell) qui se produit quand il y a recouvrement entre une région à forte densité de spin positive et une région de faible densité de spin négative résultant d'une polarisation de spin intramoléculaire.

\subsection{Transfert de charge}

Le transfert de charge concerne les composés $(A B)$ formés de deux fragments moléculaires $A$ et $B$, dans lesquels un électron est transféré de $A$ à $B$, ou de $B$ à $A$, pour former un état électronique excité $\left(A^{+} B^{-}\right)$ ou $\left(A^{-} B^{+}\right)$. Un couplage ferromagnétique peut être favorisé si le transfert de charge se produit à partir d'une orbitale de $A$ (ou de $B$ ), $a_{1}$ (ou $b_{1}$ ), occupée par un seul électron, vers une orbitale vide de $B$ (ou de $A$ ), $b_{2}$ (ou $a_{2}$ ), ou bien si chaque électron non apparié occupe des orbitales dégénérées $\left(a_{1}, a_{2}\right)$ de $A$ et $\left(b_{1}, b_{2}\right)$ de $B$. Le couplage entre l'état triplet $\left(a_{1} b_{1}\right)$ de $A B$ avec l'état triplet obtenu par transfert de charge, de plus basse énergie $\left(a_{1} a_{2}\right)$ ou $\left(b_{1} b_{2}\right)$ stabilise dans ce cas l'état fondamental triplet.

\section{DISTRIBUTION DE SPIN DANS DES RADICAUX ORGANIQUES}

Cette section est consacrée aux composés pour lesquels le magnétisme provient des électrons non appariés $2 p$ portés par des fragments organiques, appelés radicaux organiques. La diffraction de neutrons polarisés (DNP) apporte un éclairage unique sur le chemin des interactions magnétiques, dans la mesure où elle montre les atomes qui portent de la densité de spin et qui sont de ce fait impliqués dans les interactions magnétiques [21]. Le premier exemple est le biradical paramagnétique $\mathrm{Ti}(\mathrm{L})_{2}$ formé de deux radicaux semiquinonate, coordonnés à l'ion non magnétique $\mathrm{Ti}^{4+}$ et couplés de façon ferromagnétique. Le second composé, Nit-py, complètement organique, forme des chaînes ferromagnétiques de radicaux nitronyl nitroxydes reliés par des liaisons hydrogène.

\subsection{Superéchange ferromagnétique intramoléculaire entre des radicaux semiquinonate couplés par l'ion non magnétique $\mathrm{Ti}^{4+}$ dans le biradical $\mathrm{Ti}^{\mathrm{IV}}(\mathrm{Cat}-\mathrm{N}-\mathrm{SQ})_{2}$}

Le biradical $\mathrm{Ti}^{\mathrm{IV}}(\mathrm{Cat}-\mathrm{N}-\mathrm{SQ})_{2}$ est un exemple typique de couplage ferromagnétique intramoléculaire résultant de l'orthogonalité entre les orbitales magnétiques des deux radicaux Cat-N-SQ, coordonnés à un ion central $\mathrm{Ti}^{4+}\left(3 d^{0}\right)$. Le radical Cat-N-SQ est plan et a une formule chimique schématisée sur la Figure 3. L'électron non apparié est délocalisé sur le fragment moléculaire dans une orbitale $\pi$.<smiles>CC(C)(C)C1=CC(=NC2=CC(C(C)(C)C)C=C(C(C)(C)C)C2[O-])C([O-])=C(C(C)(C)C)C1</smiles>

Figure 3. Le radical Cat-N-SQ.

L'ion $\mathrm{Ti}^{4+}$ se trouve dans un environnement octaédrique formé par deux atomes d'azote en position axiale et par quatre atomes d'oxygène en position équatoriale, les plans des deux radicaux étant orthogonaux l'un par rapport à l'autre. Chaque radical est coordonné au Titane par deux liaisons OTi et une liaison N-Ti. Les propriétés magnétiques de ce composé sont celles d'un état fondamental triplet paramagnétique $(S=1)$, montrant ainsi le couplage ferromagnétique entre les spins des deux radicaux. La nature ferromagnétique de ce couplage a été clairement attribuée à l'orthogonalité des plans 
des deux radicaux, mais une incertitude demeure quant aux deux chemins possibles pour l'interaction magnétique :

- ou bien un contact direct entre les deux orbitales magnétiques aux positions des oxygènes qui se trouvent à $2.7 \AA$ l'un de l'autre

- ou bien une interaction indirecte à travers l'ion $\mathrm{Ti}^{4+}$, avec un transfert de spin des ligands vers les orbitales diffuses de $\mathrm{Ti}^{4+}$

La détermination de la carte de densité de spin a permis de choisir entre ces deux hypothèses [22]. Les mesures de neutrons polarisés ont été effectuées sur le diffractomètre 5C1 (LLB-Orphée) à une température de $1.5 \mathrm{~K}$ et sous un champ appliqué de 7 Tesla. 39 facteurs de structure ont été ainsi mesurés. Un modèle en multipôles (voir 3.2.2), limité aux termes sphériques a été utilisé pour traiter les données. Les coefficients de la partie radiale des multipôles, pris dans la littérature [17] sont reportés dans le Table 1. L'affinement des paramètres a donné un de 1.31 et un résidu $R_{W}$ de 0.16 . Les populations de spin déduites de l'affinement sont comparées dans le Table 1 aux populations de spin (populations atomiques de Mulliken) obtenues par un calcul DFT.

Table 1. Populations de spin affinées $\left(e n \mu_{B}\right.$ ) et populations de spin calculées par la méthode DFT.

\begin{tabular}{lccccc}
\hline & \multicolumn{2}{c}{ étude aux neutrons polarisés } & \multicolumn{2}{c}{ calculs DFT } \\
\hline atome & \multicolumn{2}{c}{ coefficients radiaux } & populations monopoles $\left(\right.$ en $\left.\mu_{B}\right)$ & populations de Mulliken \\
\hline & $\mathrm{n}_{l}$ & $\zeta$ a.u. $\left.^{-1}\right)$ & $\mathrm{P}_{00}$ & $2 p$ & $\mathrm{p}_{i}$ \\
\hline $\mathrm{C}$ & 2 & 3.44 & $0.22(5)$ & $2 p$ & 0.11 \\
$\mathrm{~N}$ & 2 & 3.9 & $0.20(7)$ & $2 p$ & 0.26 \\
$\mathrm{O}$ & 2 & 4.5 & $0.02(6)$ & $3 d, 3 p$ & 0.06 \\
$\mathrm{Ti}$ & 4 & 5.4 & $0.17(3)$ & & \\
\hline
\end{tabular}

La carte de densité de spin est représentée sur la Figure 4, en projection le long de la bissectrice de l'angle ${\widehat{\mathrm{O}_{1} \mathrm{TiO}}}_{2}$.

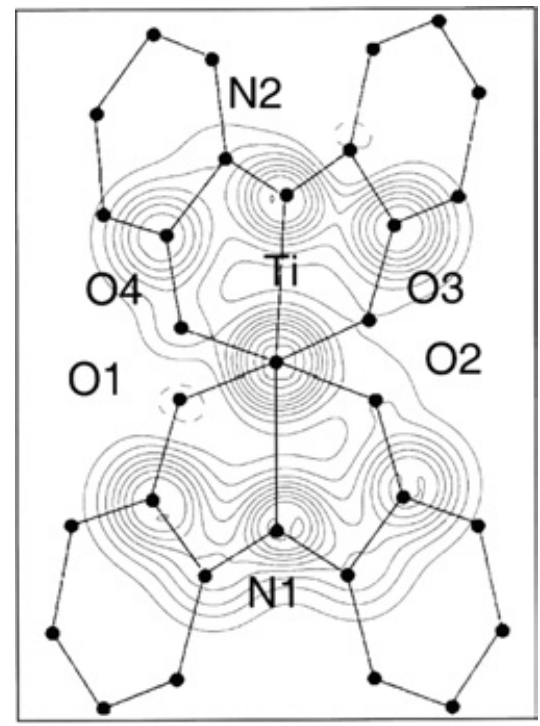

Figure 4. Carte de densité de spin de $\mathrm{Ti}^{4+}(\mathrm{Cat}-\mathrm{N}-\mathrm{SQ})_{2}$ en projection le long de la bissectrice de l'angle $\widehat{\mathrm{O}_{1} \widehat{T i O}_{2}}$. Intervalle: $0.02 \mu_{B} . \AA^{-2}$ (les contours positifs sont des lignes continues, les contours négatifs sont des lignes pointillées). 
La densité de spin est surtout délocalisée sur les atomes d'azote et sur les atomes de carbone appartenant aux quatre liaisons $\mathrm{CO}$ des radicaux, mais il n'y a pas de densité de spin sur les atomes d'oxygène. Il y a un moment magnétique de $0.17(3) \mu_{B}$ sur l'atome de titane, ce qui indique qu'un transfert de spin a lieu entre les radicaux et les orbitales diffuses $3 p$ ou $3 d$ de l'ion $\mathrm{Ti}^{4+}$. Ceci montre que l'interaction ferromagnétique est bien due à un superéchange indirect à travers les orbitales $\mathrm{Ti}^{4+}$. Les calculs DFT sont en accord qualitatif avec ces résultats, mais sous-estiment la délocalisation vers les atomes de titane et de carbone.

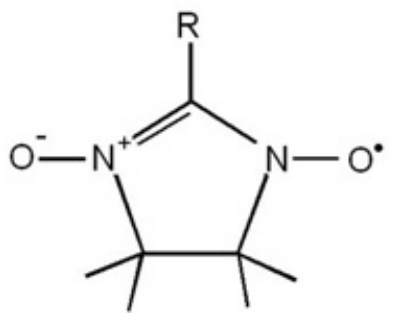

Figure 5. Le radical nitronyl nitroxyde.

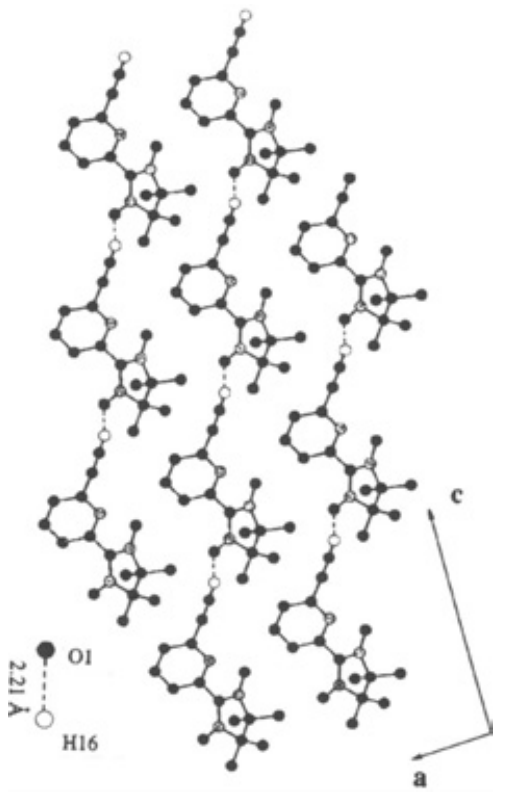

Figure 6. Structure cristalline du radical Nit-py en projection le long de l'axe $\mathbf{b}$.

\section{2 Échange ferromagnétique intermoléculaire entre des radicaux nitronyl nitroxyde Nit-Py à travers des liaisons hydrogène}

Grâce à leur potentiel de liaisons, les radicaux organiques nitronyl nitroxyde (Nit) sont des briques qui permettent d'obtenir des systèmes magnétiques de grandes dimensions. Ce radical est représenté sur la Figure 5. Un électron non apparié y est délocalisé ; il se trouve préférentiellement sur les deux 
liaisons NO, avec une distribution équilibrée entre ces quatre atomes, et peut être décrit par une orbitale moléculaire $\pi$.

Le premier radical purement organique découvert avec un ordre ferromagnétique à basse température $\left(T_{C}=0.67 \mathrm{~K}\right.$ ) est un radical nitronyl nitroxyde Nit-R où $\mathrm{R}=$ para-nitrophenyl est le groupe substituant. Ce composé a été étudié par diffraction de neutrons polarisés [23], ce qui a permis de mettre en évidence les rôles de l'orthogonalité accidentelle et les mécanismes de polarisation de spin pour les deux types d'interactions ferromagnétiques intermoléculaires.

Le composé Nit-py, où py = 2-(6-ethynyl-2-pyridyl), qui est décrit ici, forme des chaînes où les radicaux sont liés les uns aux autres par des liaisons hydrogène [24].

Un faible couplage ferromagnétique dans les chaînes a été mesuré, $J=0.9 \mathrm{~K}\left(1.4 \mathrm{~cm}^{-1}\right)$. Cependant, le composé présente un ordre antiferromagnétique entre les chaînes en dessous de $0.61 \mathrm{~K}$. Une étude de neutrons polarisés a été menée sur un échantillon monocristallin à $4.75 \mathrm{~K}$, avec un champ appliqué de 8 Tesla, sur le diffractomètre DN2 du réacteur Siloe au CEA-Grenoble (DN2 a été transporté à l'ILL et est devenu D23 après avoir subi une jouvence). Le traitement des $196 F_{M}$ expérimentaux par la méthode du Maximum d'Entropie montre à l'évidence une densité de spin positive sur l'atome $\mathrm{H}_{16}$ qui est impliqué dans la liaison hydrogène $\mathrm{N}_{2}-\mathrm{O}_{1} \cdots-\mathrm{H}_{16}$ entre deux radicaux voisins dans la chaîne [25].

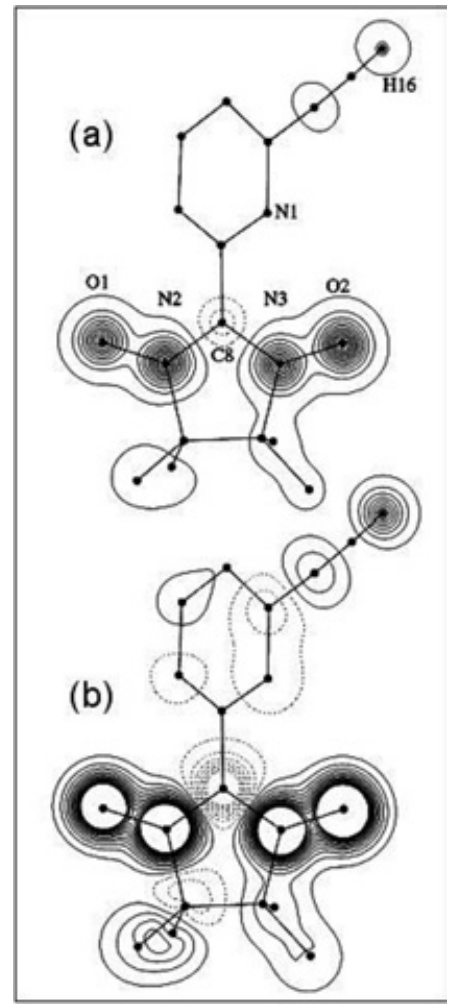

Figure 7. Densité de spin du radical Nit-py projetée sur le plan moyen du radical nitroxyde : a - contours forts (intervalle $0.04 \mu_{B} . \AA^{-2}$ ) ; b - contours faibles (intervalle $0.008 \mu_{B} . \AA^{-2}$ ).

Une carte de densité de spin a été reconstruite à partir d'un modèle dans lequel on a choisi des orbitales de type $2 p_{z}$ pour les quatre atomes des deux groupes $\mathrm{NO}$ et pour le carbone central $\mathrm{C}_{8}$, l'axe 
$z$ étant l'axe perpendiculaire au plan Nit. Pour tous les autres atomes, $y$ compris $\mathrm{H}_{16}$, on en est resté au modèle sphérique (voir 3.2.3). Les exposants pris pour les orbitales radiales étaient les suivants : $\mathrm{O}$, $\mathrm{N}, \mathrm{C}: n_{l}=2, \xi=2.25,1.95$ et 1.72 (a.u. $^{-1}$ ) et pour $\mathrm{H}, n_{l}=0$ and $\xi=2.50$ (a.u. ${ }^{-1}$ ). Les coefficients de contraction radiale ont été affinés pour $\mathrm{N}$ et $\mathrm{O}: \kappa_{N}=1.02(2)$ et $\kappa_{O}=0.90(1)$. Les résultats de l'affinement donnent un $\chi^{2}$ de 1.22. La carte de densité de spin correspondante est représentée sur la Figure 7, en projection sur le plan du Nit. Elle montre bien la délocalisation sur la molécule. La Figure 8 où la densité est projetée le long d'une ligne de ce plan, perpendiculaire à la liaison $\mathrm{NN}$, visualise bien les densités de type $2 \mathrm{pz}$ du groupe ONCNO.

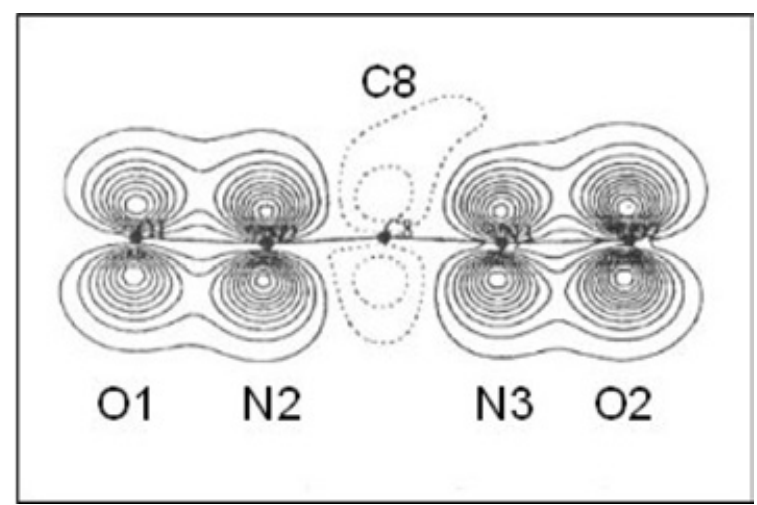

Figure 8. Densité de spin du radical Nit-py projetée sur le plan $\pi$ du nitroxyde. intervalle : $0.04 \mu_{B} . \AA^{-2}$ (les contours négatifs sont en pointillés).

Deux points importants doivent être remarqués : d'abord, on observe une population de spin positive significative sur $\mathrm{H}_{16}, P_{H_{16}}=0.04(1) \mu_{B}$, ensuite, la population de spin de l'atome $\mathrm{O}_{1}\left(P_{O_{1}}=\right.$ $\left.0.20(1) \mu_{B}\right)$, impliqué dans la liaison hydrogène, est clairement plus faible que celle de l'atome $\mathrm{O}_{2}$ $\left(P_{O_{2}}=0.28(1) \mu_{B}\right)$ de l'autre groupe NO, alors que les atomes d'azote ont des populations équivalentes $\left(P_{N_{2}}=0.24(1)\right.$ et $\left.P_{N_{3}}=0.22(1) \mu_{B}\right)$ pour les deux groupes NO. Ceci suggère que le transfert d'électron de $\mathrm{O}_{1}$ vers $\mathrm{H}_{16}$ se fait à travers la liaison hydrogène. Ces résultats vont dans le sens d'un couplage ferromagnétique entre radicaux voisins dans la chaîne par un mécanisme de transfert de charge. Des calculs DFT montrent que ce transfert de charge peut avoir lieu entre les orbitales $\pi^{\star}$ du groupe nitronyl nitroxyde vers l'orbitale non occupée la plus basse du radical voisin qui inclue une contribution de $\mathrm{H}_{16}$.

\section{DISTRIBUTION DE SPIN DANS LES COMPLEXES DE MÉTAUX DE TRANSITION}

Les composes magnétiques à base moléculaire les plus nombreux sont construits à partir de complexes organiques d'ions de transition magnétiques. Les ligands organiques sont soit des radicaux [14], portant eux-même des électrons non appariés, soit des ponts organiques qui servent de médiateurs de l'interaction magnétique entre les ions de métaux de transition [26]. Deux types de composés sont décrits dans cette section : un composé cuivre-nitroxyde formant des chaînes ferromagnétiques de spin $\frac{1}{2}$, et un complexe paramagnétique bicuivrique présentant un fort couplage intramoléculaire ferromagnétique via deux ponts azide $\mathrm{N}_{3}^{-}$. Les avantages de la technique du rapport de flipping pour l'étude des interactions magnétiques sont les suivants : - la visualisation de l'orientation des orbitales magnétiques et - la détermination du signe des densités de spin.

Un autre type d'application de la DNP (Diffraction de Neutrons Polarisés) au magnétisme à base moléculaire concerne les clusters à haut spin. La détermination de la distribution de spin dans un cluster 
paramagnétique permet d'élucider sans ambiguïté la nature de l'état fondamental de spin, alors que les mesures magnétiques macroscopiques ne donnent pas toujours une solution unique pour l'arrangement de spin.

\subsection{Chaînes Ferromagnétiques -Cu-Nit-Cu-}

Le composé $\mathrm{Cu}(\mathrm{hfac})_{2} \mathrm{NitMe}$ forme des chaînes ferromagnétiques de spin $1 / 2 \mathrm{~d}$ 'ions $\mathrm{Cu}^{2+}$ alternant avec des radicaux Nit, - $\mathrm{Cu}_{1}-\mathrm{Nit}-\mathrm{Cu}_{2}$ - Nit -, avec deux sites de cuivre dans des environnements différents [27] (Figure 9). Chacun des deux groupes NO d'un radical Nit se trouve en coordination axiale du cuivre octaédrique voisin, le plan Nit étant à peu près parallèle au plan de base du cuivre.

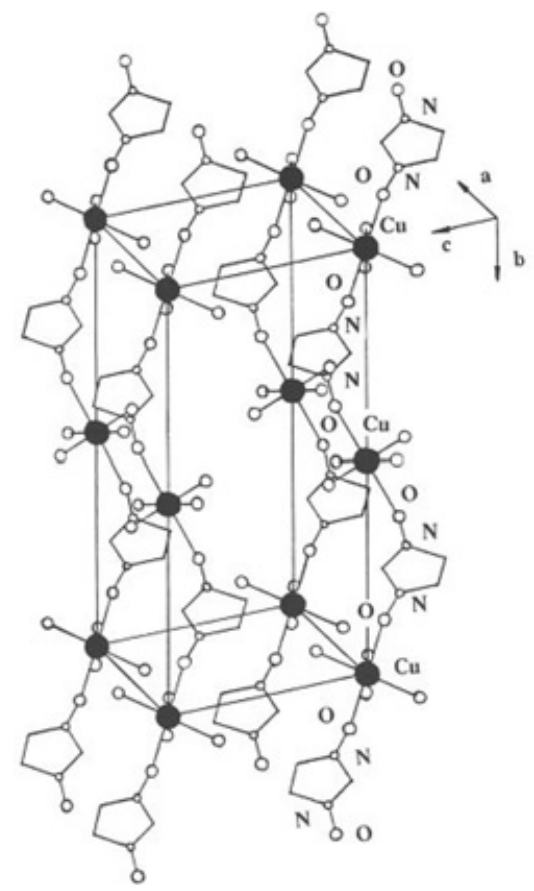

Figure 9. Contenu de la maille cristallographique pour le composé $\mathrm{Cu}(\mathrm{hfac})_{2} \mathrm{NitMe}$.

Un modèle de fonction d'onde, décrit dans la section 3.2.1, a été utilisé pour affiner la densité de spin sur 162 facteurs de structure magnétiques uniques mesurés sur D3 (ILL) à $2.5 \mathrm{~K}$ sous un champ appliqué de 4.6 Tesla. Les exposants radiaux initiaux $\xi$ (a.u. ${ }^{-1}$ ) sont les mêmes que ceux donnés dans le paragraphe (5.2) pour $\mathrm{N}, \mathrm{O}, \mathrm{C}$ et une valeur de 4.40 a.u. $^{-1}$ a été prise pour Cu d'après la littérature [17]. Les axes locaux ont été définis par rapport aux directions des liaisons: $z_{C u} / / \mathrm{Cu}-\mathrm{ON}, y_{C u} / / \mathrm{Cu}-\mathrm{O}$ (hfac) et $x_{C u} \perp(y, z)$ pour chaque atome de cuivre et $z_{N O} \perp$ Nit plane, $y_{N O} / / \mathrm{NO}, x_{N O} \perp(y, z)$ pour chaque groupe NO.

Les coefficients des orbitales $3 d$ du cuivre et des orbitales $2 s, 2 p$ centrées sur $\mathrm{N}$ et $\mathrm{O}$ ont été affinés ainsi que les populations de spin atomiques correspondantes. Pour les atomes d'oxygène appartenant au ligand hfac et les atomes de carbone du cycle Nit, des contributions sphériques à l'orbitale moléculaire ont été affinées. Les coefficients de contraction restent très proches de l'unité après affinement : $\kappa_{C u}=$ $1.06(3), \kappa_{N}=1.00(3), \kappa_{O}=1.06(5)$. Dans la Table 2 sont reportés les coefficients d'orbitales et les 
populations de spin pour les atomes de $\mathrm{Cu}, \mathrm{N}$, et $\mathrm{O}$ du radical Nit obtenus dans l'affinement final (GOF $=2.55$ ) et la carte de densité de spin expérimentale correspondante, en projection sur le plan du Nit, est présentée dans la Figure 10.
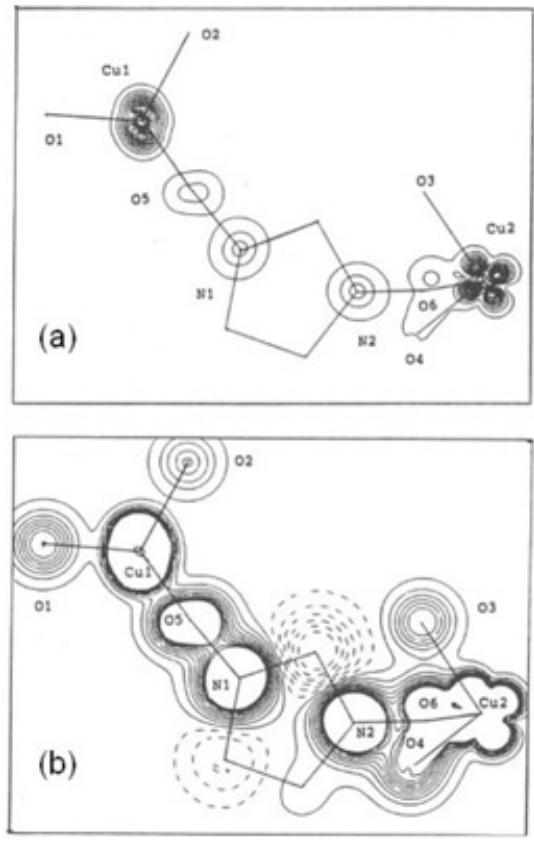

Figure 10. Densité de spin reconstruite dans $\mathrm{Cu}(\mathrm{hfac})_{2} \mathrm{NitMe}$ en projection sur le plan du Nit : (a) forts niveaux d'isodensité : intervalle $0.2 \mu_{B} . \AA^{-2}$; (b) faibles niveaux : intervalle $0.005 \mu_{B} \AA^{-2}$.

Table 2. Populations atomiques de spin $\left(\right.$ en $\left.\mu_{B}\right)$ et coefficients d'orbitales affinés pour les atomes de Cu et les groupes $\mathrm{NO}$ dans $\mathrm{Cu}(\mathrm{hfac})_{2} \mathrm{NitMe}$.

\begin{tabular}{|c|c|c|c|c|c|c|c|c|}
\hline & \multicolumn{3}{|c|}{ atomes de cuivre } & \multicolumn{5}{|c|}{ radical nitronyl nitroxyde } \\
\hline & & $\mathrm{Cu}_{1}$ & $\mathrm{Cu}_{2}$ & & $\mathrm{~N}_{1}$ & $\mathrm{~N}_{2}$ & $\mathrm{O}_{5}$ & $\mathrm{O}_{6}$ \\
\hline Coefficients & $3 d_{x^{2}-y^{2}}$ & $-0.60(17)$ & $0.91(11)$ & $2 s$ & $0.18(3)$ & $-0.04(3)$ & $-0.15(5)$ & $-0.28(6)$ \\
\hline d'orbitale & $3 d_{z^{2}}$ & $-0.33(13)$ & $0.03(16)$ & $2 p_{x}$ & $-0.40(7)$ & $-0.11(12)$ & $-0.56(13)$ & $0.77(6)$ \\
\hline atomique & $3 d_{x y}$ & $0.42(8)$ & $0.34(19)$ & $2 p_{y}$ & $-0.12(9)$ & $-0.18(8)$ & $0.29(15)$ & $-0.02(12)$ \\
\hline$a_{m}^{i}$ & $3 d_{x z}$ & $0.05(11)$ & $0.24(20)$ & $2 p_{z}$ & $0.89(3)$ & $0.98(2)$ & $0.77(6)$ & $0.57(7)$ \\
\hline & $3 d_{y z}$ & $0.59(17)$ & $0.00(15)$ & & & & & \\
\hline Population atomique & $3 d$ & $0.858(9)$ & $0.754(8)$ & $2 s$ & $0.010(3)$ & $0.001(1)$ & $0.004(3)$ & $0.015(7)$ \\
\hline de spin & & & & $2 p$ & $0.311(8)$ & $0.299(8)$ & $0.205(7)$ & $0.190(10)$ \\
\hline
\end{tabular}

La somme des populations sur chaque unité -Cu-Nit-, qui est égale à 1.89(3) $\mu_{B}$, est en accord avec la valeur de l'aimantation mesurée dans les mêmes conditions de température et de champ (1.91 $\mu_{B}$ ). Le moment total porté par le radical, $0.88 \mu_{B}$, est du même ordre de grandeur que sur chaque fragment relatif au cuivre (le cuivre et les quatre oxygènes du (hfac)) ce qui montre que les spins $1 / 2$ sont uniformément distribués sur les sites de $\mathrm{Cu}$ et Nit dans la chaîne. Dans le radical on remarque que l'atome d'azote porte une population de spin plus grande que l'atome d'oxygène de chaque groupe NO, contrairement aux radicaux nitroxydes isolés où la densité de spin est à peu près également distribuée sur $\mathrm{N}$ et $\mathrm{O}$ [28]. Un transfert de spin de l'oxygène vers l'azote accompagne donc la formation des chaînes. 
(a)

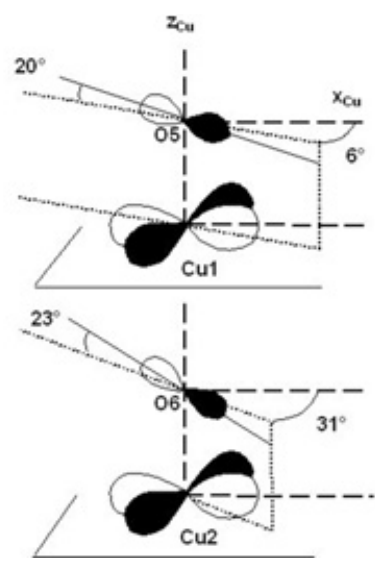

Figure 11. Orientation relative entre les orbitales magnétiques du cuivre et de l'oxygène pour les deux sites $\mathrm{Cu}_{1}$ et $\mathrm{Cu}_{2}$.

L'orbitale de $\mathrm{Cu}_{2}$ est essentiellement du type $3 d_{x^{2}-y^{2}}$ alors que l'orbitale de $\mathrm{Cu}_{1}$ apparaît plus distordue (combinaison de $3 d_{z^{2}}, 3 d_{x y}, 3 d_{y z}$ and $3 d_{x^{2}-y^{2}}$ ). Les orbitales de $\mathrm{N}_{1}$ et $\mathrm{N}_{2}$ sont principalement du type $2 p_{z}$, alors que les orbitales de $\mathrm{O}_{5}$ et $\mathrm{O}_{6}$ ont des coefficients importants sur $2 p_{x}$ et $2 p_{z}$. Ceci correspond à un tilt de l'orbitale $2 p$ de l'oxygène vers la direction $x$, perpendiculaire à la liaison $\mathrm{NO}$, qui se trouve dans le plan du Nit et presque dans le plan de base du cuivre voisin. On peut voir sur la carte de densité de spin dans la Figure 10 que l'orientation de l'orbitale de l'oxygène $\mathrm{O}_{6}$ est presque parallèle au plan de base du $\mathrm{Cu}_{2}$. Ceci s'accompagne d'une contribution significative $2 s$ reflétant une hybridation de l'atome d'oxygène coordonné au cuivre. La Figure 11 schématise l'orientation relative entre l'orbitale $2 p$ de l'oxygène et l'orbitale $3 d$ pour chaque site $\mathrm{Cu}_{1}$ et $\mathrm{Cu}_{2}$, déduite de l'affinement de densité de spin.

L'intégrale de recouvrement entre les orbitales magnétiques du cuivre et de l'oxygène est minimisée par l'orientation relative des orbitales qui font des angles de 20 degrés seulement entre la direction $2 p$ et le plan de l'orbitale $3 d$. Le mécanisme de couplage ferromagnétique est donc consistant avec le schéma d'orthogonalité accidentelle.

\subsection{Couplage Intramoléculaire $\mathrm{Cu}-\mathrm{Cu}$ ferromagnétique via des groupes 'end-on' $\mathrm{N}_{3}^{-}$pontants}

Le pont azide $\mathrm{N}_{3}^{-}$occupe une place particulière dans la large variété de ponts organiques utilisés en magnétisme moléculaire. Pour tous les dimères symétriques di- $\mu$-azido-cu(II) synthétisés jusqu'à présent, on observe soit un fort couplage ferromagnétique, soit un fort couplage antiferromagnétique, selon la manière dont les deux groupes azide pontent les cuivres, soit par une extrémité, 'end-on' $(\mu-1,1-)$, soit par les deux extrémités, 'end-to-end' $(\mu-1,3-)$. Jusqu'à récemment, une longue controverse a eu lieu sur l'origine du fort couplage ferromagnétique dans les dimères 'end-on' de composés di- $\mu$-azide cu(II) [29]. Les deux mécanismes principaux invoqués sont :

- le mécanisme de délocalisation de spin responsable de l'orthogonalité accidentelle des orbitales magnétiques du cuivre délocalisées sur les atomes d'azote pontants: le signe de la constante de couplage dépend alors de la valeur de l'angle $\mathrm{CuNCu}[30]$,

$$
J\left(\mathrm{~cm}^{-1}\right)=-C \alpha(\text { deg. })+D
$$

où $C$ and $D$ sont des valeurs empiriques pour une série de composés de la même famille.

- le mécanisme de polarisation de spin, qui implique la présence d'une densité de spin négative sur les atomes pontants et prédit un couplage ferromagnétique quelque soit l'angle du pont $\mathrm{CuNCu}$. 
Parce que ces deux mécanismes impliquent des signes contradictoires pour la densité de spin sur les azotes pontants, la détermination expérimentale de la densité de spin fournit un test décisif. Les mesures de neutrons polarisés ont été effectuées sur D3 à l'ILL sur le composé moléculaire 'end-on' $\mathrm{Cu}_{2}$ (t-bupy) $)_{4}\left(\mathrm{~N}_{3}\right)_{2} \cdot\left(\mathrm{ClO}_{2}\right)_{2}$ (avec t-bupy = p-tert-butylpyridine) qui présente un large gap singulet-triplet de $105 \mathrm{~cm}^{-1}$ entre l'état fondamental triplet paramagnétique $(\mathrm{S}=1)$ et l'état singulet excité [31]. Un jeu de 152 facteurs de structure magnétiques $(F>3 \sigma)$ a été obtenu à $1.6 \mathrm{~K}$ sous un champ appliqué de 4.6 Tesla, après correction de la contribution orbitale du cuivre en utilisant l'approximation dipolaire avec $g_{C u}=2.15$. La méthode du maximum d'entropie a montré qu'une région de densité de spin positive se situe sur la position de l'azote pontant. L'affinement d'un modèle de densité de spin a été effectué utilisant le modèle général décrit dans la section 3.2.3. Les coefficients $n$ and $\zeta$ pour la densité radiale sont : $n=4, \zeta=8.8$ a.u. $^{-1}$ pour $\mathrm{Cu}, n=2, \zeta=3.9$ a.u. ${ }^{-1}$ pour $\mathrm{N}$ et $\zeta=3.44$ a.u. ${ }^{-1}$ pour C. Un coefficient de contraction a été affiné pour $\mathrm{Cu}$ et $\mathrm{N}\left(\kappa_{C u}=1.10(1)\right.$ et $\left.\kappa_{N}=1.90(1)\right)$. Des populations de monopôles ont été affinées pour les atomes $\mathrm{N}$ et $\mathrm{C}$ des cycles pyrimidine. Différents types de contraintes orbitalaires ont été testés pour les atomes de $\mathrm{Cu}$ et d'azote du groupe azide. Les meilleurs facteurs d'agrément, $R_{W}(F)=0.065$ et $\mathrm{GOF}=1.45$, ont été obtenus en appliquant une contrainte du type $3 d_{x y}$ or $3 d_{x^{2}-y^{2}}$ sur le cuivre (c'est-à-dire dans le plan de base) et px sur l'azote terminal du groupe azide ( $x$ étant $/ / \mathrm{Cu}-\cdots-\mathrm{Cu}$ ). Les populations atomiques de spin affinées, normalisées à $2 \mu_{B}$, sont reportées dans le Table 3.

Table 3. Populations atomiques de spin expérimentales et théoriques DFT.

\begin{tabular}{lcclc}
\hline atome & \multicolumn{3}{c}{ populations de spin } \\
\hline \multicolumn{4}{c}{ neutrons } & \multicolumn{2}{c}{ DFT (Mulliken) } \\
\hline $\mathrm{Cu}$ & $3 d_{x^{2}-y^{2}}$ & $0.783(7)$ & $3 d$ & 0.425 \\
$\mathrm{~N}_{1}$ & $P_{00}$ & $0.069(6)$ & $2 p$ & 0.167 \\
$\mathrm{~N}_{2}$ & $P_{00}$ & $-0.016(6)$ & $2 p$ & -0.005 \\
$\mathrm{~N}_{3}$ & $2 p_{x}$ & $0.057(7)$ & $2 p$ & 0.122 \\
$\mathrm{~N}_{4}$ & $P_{00}$ & $0.067(8)$ & $2 p$ & 0.129 \\
$\mathrm{~N}_{5}$ & $P_{00}$ & $0.049(7)$ & $2 p$ & 0.120 \\
\hline
\end{tabular}

Le spin est essentiellement localisé sur les ions de cuivre mais une densité de spin positive est observée sur les atomes d'azote pontants et terminaux des groupes azides ainsi que sur les azotes des cycles pyridiniques. La carte de densité de spin induite est représentée dans la Figure 12.

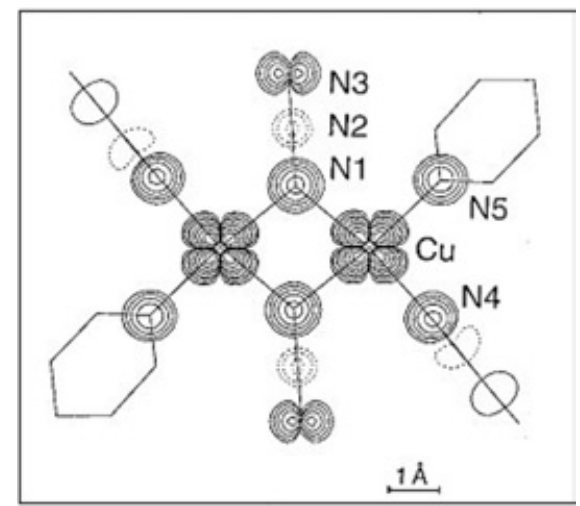

Figure 12. Carte de densité de spin dans $\mathrm{Cu}_{2}(\mathrm{t} \text {-bupy })_{4}\left(\mathrm{~N}_{3}\right)_{2} \cdot\left(\mathrm{ClO}_{2}\right)_{2}$ en projection sur le pont central. Niveaux d'isodensité logarithmiques : $\pm 0.005 \times 2^{n-1} \mu_{B} . \AA^{-2}$. 
Le signe positif sur l'azote pontant exclut définitivement le mécanisme de polarisation de spin et démontre que la distribution de spin est principalement due à un mécanisme de délocalisation de spin. Toutefois, la carte de densité de spin révèle également l'existence d'une densité négative sur l'atome d'azote central $\mathrm{N}_{2}$, qui peut être attribuée à la polarisation de spin de l'orbitale doublement occupée de type $\pi_{g}$ du pont azide, qui se superpose au mécanisme de délocalisation de spin. Les populations de spin calculées par la méthode DFT-B3LYP utilisant le programme Gaussian 94 pour l'état fondamental triplet sont données dans le Table 3. Cette méthode prédit un état fondamental singulet pour un angle $\alpha$ plus grand que 104 degrés en accord avec le mécanisme de délocalisation de spin. La délocalisation de spin est comme toujours surestimée par les calculs DFT dans les composés contenant du cuivre, mais l'alternance de spin sur le pont azide, due à la polarisation de spin, est en accord avec l'expérience.

\subsection{Nature de l'état fondamental de spin dans un cluster à haut spin $\mathrm{Fe}_{8}^{\mathrm{III}}$}

Le cluster $\mathrm{Fe}_{8} \mathrm{Br}_{8},\left\{\left[(\operatorname{tacn})_{6} \mathrm{Fe}_{8} \mathrm{O}_{2}(\mathrm{OH})_{12}\right] \mathrm{Br}_{7} \cdot \mathrm{H}_{2} \mathrm{O}\right\}\left[\mathrm{Br} .8 \mathrm{H}_{2} \mathrm{O}\right]$, (avec tacn = 1,4,7-triazacyclono-nane) a attiré un fort intérêt dans ces dernières années en raison de l'observation de l'effet tunnel quantique de l'aimantation [3]. Une autre propriété remarquable est la forte anisotropie magnétique du cluster, bien que chaque ion $\mathrm{Fe}^{\mathrm{III}}(\mathrm{S}=5 / 2)$ soit individuellement isotrope. Ce cluster, formé par huit ions $\mathrm{Fe}^{\mathrm{III}}$ reliés par des ponts oxo et hydroxo, a une structure dite en 'papillon'. Un modèle avec six atomes de Fer portant un spin 'up' et deux avec un spin 'down' a été proposé à partir des mesures de susceptibilité magnétique, pour la configuration de spin de l'état fondamental magnétique $S=10$, résultant de la compétition entre les diverses interactions antiferromagnétiques intra-cluster.

Afin de déterminer la nature exacte de l'état fondamental de spin, la densité d'aimantation a été déterminée par diffraction de neutrons polarisés sur D3 à l'ILL pour le cluster $\mathrm{Fe}_{8} \mathrm{Br}_{8}$, qui cristallise dans le groupe d'espace non centro-symétrique P1 [32]. La densité d'aimantation induite à $2 \mathrm{~K}$ sous un champ de $4.6 \mathrm{~T}$ présentée dans la Figure 13 a été reconstruite grâce à la méthode MaxEnt appliquée à des structures non centro-symétriques [13].

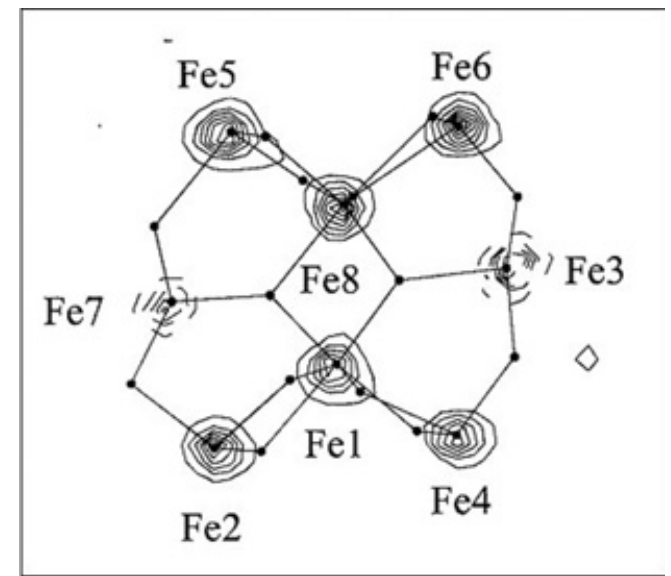

Figure 13. Densité de spin MaxEnt dans $\mathrm{Fe}_{8} \mathrm{Br}_{8}$, en projection sur le plan (b,c) (contours négatifs en pointillés, intervalle $0.7 \mu_{B} . \AA^{-2}$ ).

Un modèle de densité de spin a ensuite été affiné sur la base des 198 rapports de flipping expérimentaux. Ce modèle consiste en une somme de densités de spin atomiques sphériques sur les sites de Fer, avec des exposants radiaux $\zeta_{\mathrm{Fe}}=7.46$ a.u. $^{-1}$ [17]. Un coefficient de contraction radial commun $\kappa_{\mathrm{Fe}}$ a été affiné pour les atomes de Fer : $\kappa_{F e}=0.499(1)$. La carte représentée dans la Figure 13 confirme 
que six ions portent des spins parallèles au champ appliqué, c'est à dire les ions 1 and 2 du corps du papillon et les quatre ions périphériques, alors que les ions 3 and 4 des ailes portent des spins opposés, en accord avec le modèle proposé précédemment. Toutefois, la distribution de spin s'écarte fortement de la symétrie D2 qui peut être assumée au regard de la géométrie moléculaire: les populations de spin des ions $\mathrm{Fe}_{1}\left[4.79(22) \mu_{B}\right]$ et $\mathrm{Fe}_{2}\left[2.34(26) \mu_{B}\right]$ du corps sont très différents l'un de l'autre, ainsi que les populations des fers sur les ailes $\mathrm{Fe}_{3}\left[-1.94(31) \mu_{B}\right]$ et $\mathrm{Fe}_{4}\left[-4.91(32) \mu_{B}\right]$ entre elles. Ceci pourrait être dû à l'existence d'interactions magnétiques intermoléculaires, les atomes de Fer correspondants étant dans des environnements différents.

Afin de vérifier si cette dissymétrie est significative ou seulement due à un artefact relié au cas particulier d'un groupe d'espace non centro, une expérience de diffraction de neutrons polarisés a été récemment effectuée sur $5 \mathrm{C} 1$ au LLB sur un cluster centro-symétrique $\mathrm{Fe}_{8} \mathrm{pcl}$ [33]. Ce composé, de formule $\left[(\operatorname{tacn})_{6} \mathrm{Fe}_{8} \mathrm{O}_{2}(\mathrm{OH})_{12}\right] \mathrm{Br}_{4.3}\left(\mathrm{ClO}_{4}\right)_{3.7} \cdot 6 \mathrm{H}_{2} \mathrm{O}$, présente essentiellement le même coeur central que $\mathrm{Fe}_{8} \mathrm{Br}_{8}$, mais avec un centre de symétrie, et cristallise dans le groupe d'espace centro-symétrique $\mathrm{P} 2{ }_{1} / \mathrm{c}$ avec deux molécules de cluster par maille [34]. Les propriétés magnétiques de Fe8pcl sont très similaires à celles de $\mathrm{Fe}_{8} \mathrm{Br}_{8}$ avec un état fondamental de spin $S=10$.

Un modèle sphérique de densité de spin similaire à celui défini pour $\mathrm{Fe}_{8} \mathrm{Br}_{8}$ a été affiné sur la base de 52 facteurs de structure magnétiques expérimentaux avec $F_{M}>\sigma(\mathrm{T}=1.8 \mathrm{~K}, \mathrm{H}=7 \mathrm{~T})$. Un facteur d'accord pondéré $R_{W}(F)=0.104$ et un facteur de qualité $\chi=2.31$ ont été obtenus. La densité de spin localisée sur les atomes de Fer dans $\mathrm{Fe}_{8}$ pcl est moins diffuse que dans $\mathrm{Fe}_{8} \mathrm{Br}_{8}$ comme l'indiquent les coefficients de contraction radiaux différents : $\kappa_{F e}=0.71(3)$ dans $\mathrm{Fe}_{8} \mathrm{pcl}$ par rapport à $\kappa_{F e}=0.499(1)$ dans $\mathrm{Fe}_{8} \mathrm{Br}_{8}$. La somme des populations affinées est égale à 17.5(4) $\mu_{B}$ c'est à dire 92 pour cent de la valeur de l'aimantation expérimentale (égale à $19 \mu_{B}$ à $1.8 \mathrm{~K}$ sous 7 Tesla). La carte de densité de spin correspondante est représentée en projection selon la perpendiculaire au plan du corps du papillon dans la Figure 14.

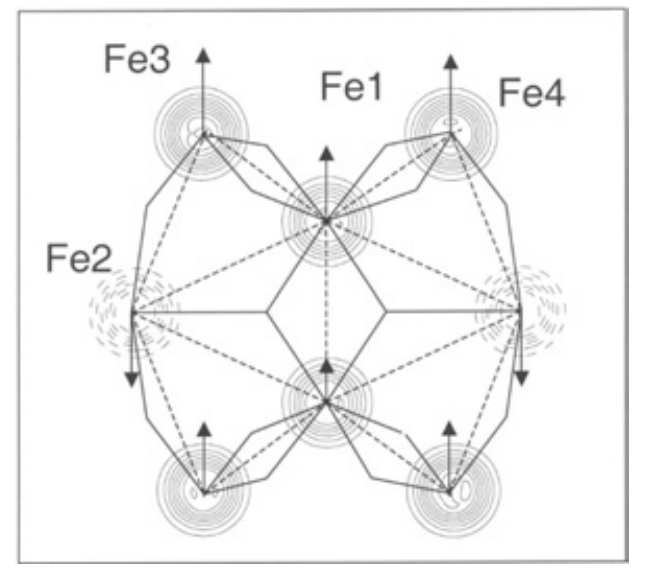

Figure 14. Projection de la densité de spin induite à $1.8 \mathrm{~K}$ sous $7 \mathrm{~T}$ dans $\mathrm{Fe} 8 \mathrm{pcl}$ selon la perpendiculaire au plan du corps du papillon. Contours de faible isodensité $: \pm 0.5 \mu_{B} . \AA^{-2}$ avec intervalles de $0.5 \mu_{B} . \AA^{-2}$. La structure du c?ur et les chemins d'interaction magnétiques sont schématisés sur la carte.

L'arrangement relatif des spins est en accord avec l'étude précédente de densité de spin dans $\mathrm{Fe}_{8} \mathrm{Br}_{8}$ avec des populations de spin positives sur $\mathrm{Fe}_{1}, \mathrm{Fe}_{3}$ et $\mathrm{Fe}_{4}$ et négatives sur $\mathrm{Fe}_{2}$. Les populations de spin des ions du corps $\mathrm{Fe}_{1}$ et $\mathrm{Fe}_{2}$ en valeur absolue (environ $3 \mu_{B}$ ) sont plus faibles que les populations des ions $\mathrm{Fe}_{3}$ et $\mathrm{Fe}_{4}$ des ailes (environ $4 \mu_{B}$ ). Il est à remarquer que les populations de spin sur $\mathrm{Fe}_{1}$ et $\mathrm{Fe}_{2}$ dans $\mathrm{Fe}_{8}$ pcl correspondent respectivement à la valeur moyenne des populations de spin $\left(\mathrm{Fe}_{1}, \mathrm{Fe}_{2}\right)$ 
et $\left(\mathrm{Fe}_{3}, \mathrm{Fe}_{4}\right)$ observées dans le composé non centro $\mathrm{Fe}_{8} \mathrm{Br}_{8}$. C'est pourquoi la dissymétrie de la carte de densité de spin dans le cas non centro est sans doute plutôt due au traitement des données qu'à la dissymétrie des interactions magnétiques intermoléculaires pour des environnements différents.

\section{APPLICATION À UN COMPOSÉ MOLÉCULAIRE PHOTO-COMMUTABLE}

Les composés à transition de spin contenant l'ion $\mathrm{Fe}^{2+}$ ion peuvent être commutés en variant la température ou la pression, ou par irradiation lumineuse, d'un état bas spin (BS) diamagnétique $(S=0)$ et un état haut spin (HS) paramagnétique $(S=2)$ et vice versa, comme cela est schématisé dans la Figure 15.

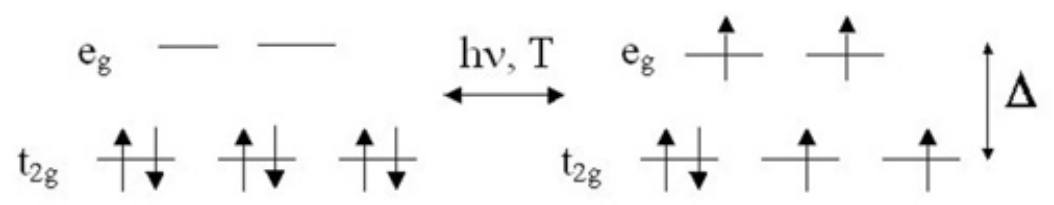

Figure 15. Transition entre l'état fondamental $S=0$ et l'état excité $S=2$ dans un complexe de $\mathrm{Fe}^{2+}$.

La photo-excitation à basse température, par une lumière de longueur d'onde adéquate, induit une transition du système dans l'état fondamental bas spin BS vers un état HS métastable ayant une extrêmement longue durée de vie à basse température. C'est pourquoi cet effet est appelé Light Induced Excited Spin State Trapping (LIESST) [35]. L'application de la DNP à l'étude de l'état magnétique photo-induit du composé à transition de spin $\left[\mathrm{Fe}(\mathrm{ptz})_{6}\right]\left(\mathrm{BF}_{4}\right)_{2}(\mathrm{ptz}=1$-propyltetrazole) est décrite dans cette section.

Le processus de photo-commutation a été observé en utilisant un nouveau montage expérimental permettant à la fois l'irradiation lumineuse et les mesures de diffraction de neutrons polarisés (DNP) [36] comme le montre le schéma (Figure 16).

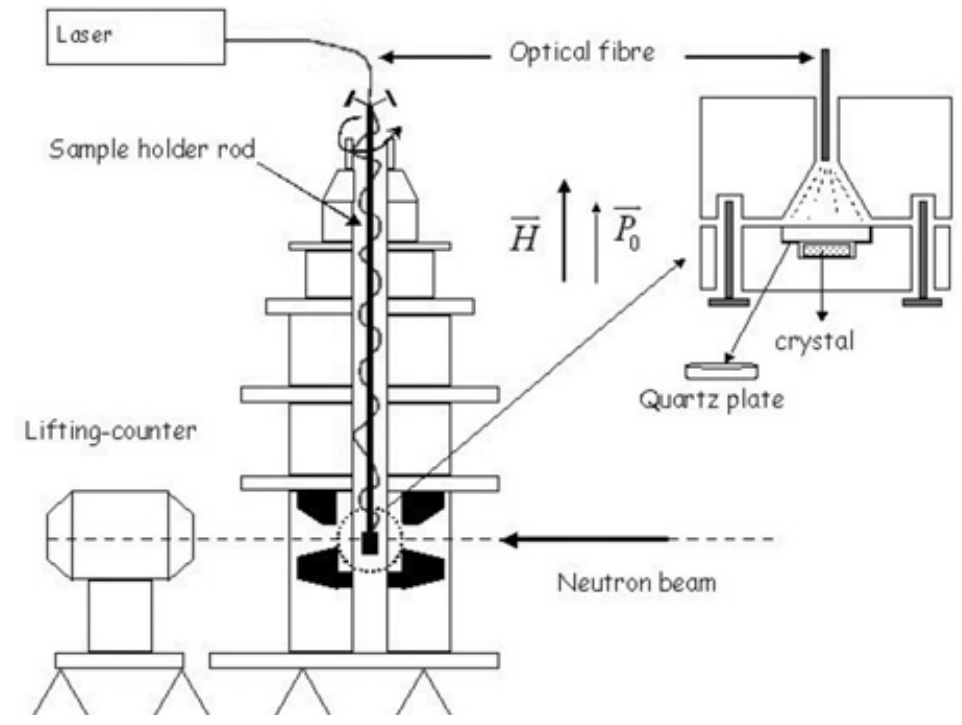

Figure 16. Dispositif instrumental pour la photo-excitation in-situ sur $5 \mathrm{C} 1$. 
Le composé $\left[\mathrm{Fe}(\mathrm{ptz})_{6}\right]\left(\mathrm{BF}_{4}\right)_{2}$ cristallise dans le groupe d'espace trigonal $R \overline{3}$. Au cours d'un refroidissement lent, le composé $\left[\mathrm{Fe}(\mathrm{ptz})_{6}\right]\left(\mathrm{BF}_{4}\right)_{2}$ subit une transition de spin thermique de la phase haute température (HS) vers la phase basse température (BS), avec $T_{C}^{\downarrow}=128 \mathrm{~K}$ et $T_{C}^{\dagger}=135 \mathrm{~K}$, à pression ambiante, accompagnée par une transition de phase cristallographique vers $130 \mathrm{~K}$ de $R \overline{3}$ vers $\mathrm{P} 1$ à basse température. En refroidissant rapidement, la transition de phase cristallographique peut être supprimée, de sorte que le cristal conserve le groupe d'espace $R \overline{3}$ à basse température. Le cristal a ainsi été d'abord plongé dans l'azote liquide puis placé dans le cryoaimant installé sur le diffractomètre $5 \mathrm{C} 1$, et ensuite refroidi à $2 \mathrm{~K}$. Le champ magnétique a été appliqué selon l'axe c. Le cristal a été éclairé par un faisceau laser de puissance $3 \mathrm{~mW}$ à la longueur d'onde $\lambda=473 \mathrm{~nm}$. La direction de la lumière était perpendiculaire à la surface du cristal.

La dépendance du processus de photo-excitation du cristal en fonction du temps a été directement suivi par DNP dans un champ constant de 5 T. Pour cela, le rapport de flipping $R$ de la raie $(0,1$, 2) a été mesuré en fonction du temps. Afin d'éviter le chauffage de l'échantillon par le faisceau laser, une expérience de lumière hachée a été effectuée. L'évolution de $(1-R)$ en fonction du temps sous illumination laser à $2 \mathrm{~K}$ est présentée dans la Figure 17.

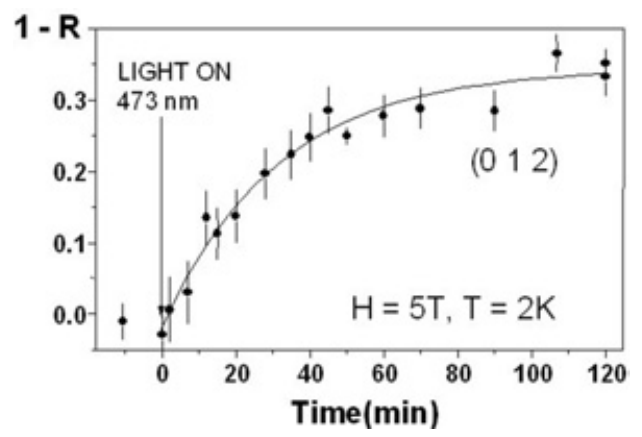

Figure 17. Cinétique du processus de photo-excitation à $2 \mathrm{~K}$, sous $5 \mathrm{~T}$, pour une irradiation à $473 \mathrm{~nm}$ de puissance $30 \mathrm{~mW}$.

La dépendance en température de l'aimantation induite par un champ de $5 \mathrm{~T}(\mathbf{H} / / \mathbf{c})$ à $2 \mathrm{~K}$ dans l'état photo-excité du cristal de $\left[\mathrm{Fe}(\mathrm{ptz})_{6}\right]\left(\mathrm{BF}_{4}\right)_{2}$ a été mesurée par DNP et SQUID. Un bon accord entre les deux techniques a été obtenu [36]. La relaxation l'état photo-excité vers l'état électronique, due à l'activation thermique du processus de relaxation $\mathrm{HS} \longrightarrow \mathrm{BS}$, a été observée dans le domaine de température $55-60 \mathrm{~K}$.

La distribution de densité d'aimantation dans la maille a été reconstruite à partir de 67 facteurs de structure magnétiques par affinement d'un modèle sphérique. L'extension radiale et les populations de monopôles ont été affinés pour le Fer et les atomes d'azote premiers voisins et un facteur de qualité $G O F=2.26$ a été obtenu. La projection de la densité d'aimantation induite selon l'axe cristallographique c est présentée dans la Figure 18.

Aucune densité significative n'a été observée dans l'affinement sur les atomes d'azote $(0.03(8)$ $\left.\mu_{B}\right)$. Le moment de 4.05(7) $\mu_{B}$ sur le site de Fer est très proche de la valeur théorique du moment de l'ion $\mathrm{Fe}^{2+}$ à saturation $(S=2)$. Ceci fournit une mise en évidence directe de la complète phototransformation du cristal.

Cette première détermination de la densité d'aimantation dans un état excité ouvre de nouvelles perspectives d'investigations dans le domaine du photo-magnétisme. Dans les composés à transfert de charge où la photo-excitation induit un transfert d'électron d'un site magnétique vers un autre, modifiant les états locaux de spin, une mise en évidence directe de ce mécanisme de transfert pourrait ainsi être donnée par l'étude de la densité d'aimantation dans l'état fondamental et dans l'état excité. 


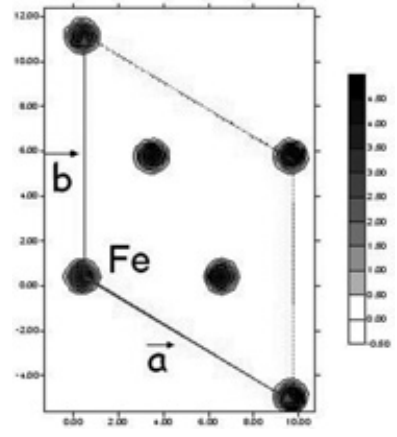

Figure 18. Carte de densité d'aimantation induite dans l'état photo-excité, à $2 \mathrm{~K}$, sous 5 Tesla, du compose à transition de spin $\left[\mathrm{Fe}(\mathrm{ptz})_{6}\right]\left(\mathrm{BF}_{4}\right)_{2}$, en projection selon l'axe c.

\section{REMERCIEMENTS}

L'auteur remercie J. Schweizer pour la traduction française de ce document ainsi qu'E. Ressouche et Y. Pontillon.

\section{Références}

[1] Kahn, O., in 'Molecular Magnetism', VVH Publishers, Inc., New York (1993).

[2] Ferlay, S., Mallah, T., Ouahès, R., Veillet, P., Verdaguer, M., Nature 378 (1995) 701.

[3] Delfs, C., Gatteschi, D., Pardi, L., Sessoli, R., Wieghard, K., Hanke, D., Inorg. Chem. 32 (1993) 3099.

[4] Schweizer, J., in 'Introduction to physical techniques in molecular magnetism', Proc. of the Yesa Summer School, Ed. by Palacio, F., Ressouche, E., Schweizer, J., Zaragoza, 2000.

[5] Bonnet, M., Delapalme, A., Becker, P., Fuess, H., Acta Cryst. A 32 (1976) 945.

[6] Marshall, W. and Lovesey, S. W., Theory of Thermal Neutron Scattering, Oxford Univ. Press, Oxford 1971.

[7] Ressouche, E., Thèse, Univ. Grenoble I (1991).

[8] Forsyth, J. B., Wells, M., Acta Cryst 12 (1959) 412.

[9] Squires, G. L., in Introduction to the theory of thermal neutron scattering (Cambridge : University Press, 1978), p. 139.

[10] Brown, P. J., Magnetic form factors, chapter 4.4.5, International tables for crystallography (1992) vol. C (A. J. C. Wilson, ed.), pp. 391-399.

[11] Boucherle, J.X., Gillon, B., Maruani, J., Schweizer, J., Mol. Phys. 60 (1987) 1121.

[12] Papoular, R. and Gillon, B., Europhys. Lett. 13 (1990) 429.

[13] Schleger, P., Puig-Molina, A., Ressouche, E., Schweizer, J., Acta Cryst. A53 (1997) 426.

[14] Schweizer, J., Ressouche, E., in 'MagnetoScience Đ From Molecules to Materials', Eds Miller, J., Drillon, M., Wiley, (2001) pp. 325-355.

[15] Brown, P. J., Capiomont, A., Gillon, B., Schweizer, J., J. Mag. Mag. Mat. 14 (1979) 289; Gillon, B., Thèse d'Etat, Univ. Pierre et Marie Curie, Paris (1983).

[16] Hansen, N. K. and Coppens, P., Acta Cryst A34 (1978) 909.

[17] Clementi, E. and Raimondi, L. D., J. Chem. Phys. 38 (1963) 2686; Clementi, E. and Roetti, C., Atomic Data and Nuclear Data Tables 14 (1974) 177; Roetti, C. and Clementi, E., J. Chem. Phys. 60 (1974) 4725. 
[18] Holladay, A., Leung, P., Coppens, P., Acta Cryst A39 (1983) 377.

[19] Brown, J., Cambridge Crystallographic Subroutine Library (2006).

[20] Kollmar, C. and Kahn, O., Acc. Chem. Res. 26 (1993) 259.

[21] Schweizer, J., Gillon, B., in 'Magnetic properties of organic radicals', Ed. P.M. Lahti, Marcel Dekker, Inc., New York (1999) pp. 449-473.

[22] Pontillon, Y., Bencini, A., Caneschi, A., Dei, A., Gattteschi, D., Gillon, B., Sangregorio, C., Stride, J., Totti, F., Angew. Chem. Int. Ed. 39 (2000) 1786.

[23] Zheludev, A., Bonnet, M., Ressouche, E., Schweizer, J., Wan, M., Wang, H., J. Mag. Mag. Mat. 135 (1994) 147.

[24] Romero, F. M., Ziessel, R., De Cian, A., Fischer, J., Turek, P., New J. Chem. 20 (1996) 919.

[25] Romero, F. M., Ziessel, R., Bonnet, M., Pontillon, Y., Ressouche, E., Schweizer, J., Delley, B., Grand, A., Paulsen, C., J. Am. Chem. Soc. 122 (2000) 1298.

[26] Gillon, B., in 'MagnetoScience : From Molecules to Materials', Eds Miller, J., Drillon, M., Wiley, (2001) pp. 357-378.

[27] Ressouche, E., Boucherle, J. X., Gillon, B., Rey, P., Schweizer, J., J. Am. Chem. Soc. 115 (1993) 3610.

[28] Brown, P. J., Capiomont, A., Gillon, B., Schweizer, J., Mol. Phys. 48 (1983) 753.

[29] Sikorav, S., Bkouche-Wacksman, I., Kahn, O., Inorg. Chem. 33 (1984) 490.

[30] Thomson, L. K., Tandon, S.S., Manuel, M. E., Inorg. Chem. 34 (1995) 2356.

[31] Aebersold, M. A., Gillon, B., Plantevin, O., Pardi, L., Kahn, O., Bergerat, P., von Seggern, I., Tuczek, F., Öhrström, L., Grand, A., Lelièvre-Berna, E., J. Am. Chem. Soc. 120 (1998) 5238.

[32] Pontillon, Y., Caneschi, A., Gatteschi, D., Sessoli, R., Ressouche, E., Schweizer, J., Lelièvre-Berna, E., J. Am. Chem. Soc. 121 (1999) 5342.

[33] Sangregorio, C., Gillon, B., Pontillon, Y., Ressouche, E., Sessoli, R., Caneschi, A., Gatteschi, D., to be published.

[34] Sangregorio, C., Ohm, T., Paulsen, C., Sessoli, R., Gatteschi, D., Phys. Rev. Lett. 78 (1997) 4645.

[35] Decurtins, S., Gütlich, P., Hasselbach, M. K., Spiering, H., Hauser, A., Inorg. Chem. 24 (1985) 2174.

[36] Goujon, A., Gillon, B., Gukasov, A., Jeftic, J., Nau, Q., Codjovi, E., Varret, F., Phys. Rev. B 67 (2003) 220401 